"This document is the Accepted Manuscript version of a Published Work that appeared in final form in JOC, copyright (C) American Chemical Society after peer review and technical editing by the publisher. To access the final edited and published work see [insert ACS Articles on Request authordirected link to Published Work, see http://pubs.acs.org/doi/abs/10.1021/acs.joc.6b01118."

\title{
Iodine(III)-Mediated Selective Intermolecular C-H Amination for the \\ Chemical Diversification of Tryptamines
}

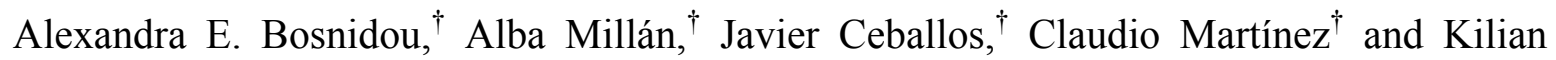
Muñiz*t:

Institute of Chemical Research of Catalonia (ICIQ), The Barcelona Institute of Science and Technology, Av. Països Catalans 16, E-43007 Tarragona (Spain)

ICREA, Pg. Lluís Companys 23, 08010 Barcelona (Spain)

Corresponding Autor. Email: kmuniz@iciq.es

\section{Abstract Graphic/ToC}<smiles>[R]C1=NCCC2(O1)C(=O)Nc1ccccc12</smiles>

\section{Abstract}


Defined hypervalent iodine reagents of the general structure $\left.\mathrm{PhI}\left[\mathrm{N}\left(\mathrm{SO}_{2} \mathrm{R}\right) \mathrm{SO}_{2} \mathrm{R}^{\prime}\right)\right]_{2}$ promote the selective direct $\mathrm{C}-\mathrm{H}$-amination of the indole core of various tryptamines. Starting from a general C2-amination strategy, subsequent transformations enable a variety of site-selective functionalizations, which proceed with noteworthy high chemoselectivity and provide an overall access to structurally diversified products.

\section{Introduction}

Hypervalent iodine(III) reagents have emerged as versatile tools in modern organic oxidation, where they have contributed to the realization of new chemical transformations. ${ }^{1,2}$ The ability of hypervalent iodine(III) reagents to expand synthetic possibilities and their benign reactivity without requirement for metal promoters have stirred particular interest from fields such as pharmaceutical and medicinal chemistry. In these cases, the supply of suitable molecules for biological studies prefers transformations that do not lead to potential contamination by metal-traces.

We have recently been interested in the application of iodine(III) compounds as reagents or catalysts for the synthesis of indoles. ${ }^{3}$ Within the quest for new oxidative functionalization of indoles, hypervalent iodine reagents have also been employed with certain success in recent years. In particular, (diacetoxy)iodosobenzene $\left(\mathrm{PhI}(\mathrm{OAc})_{2}\right)$ has enabled interesting transformations such as the direct $\mathrm{C} 3$-oxygenation ${ }^{4}$ and trans-diacetoxylation $^{5}$ of $\mathrm{N}$ protected indoles, respectively. Application of alternative hypervalent iodine(III) reagents should provide access to complementary substitution at the indole core. Within this concept, Ackermann disclosed the direct C3-arylation of indoles with diaryliodonium salts (Scheme 1, eq. 1). ${ }^{6}$ 


\section{Iodine-Mediated Direct 3-Arylation of Indoles:}

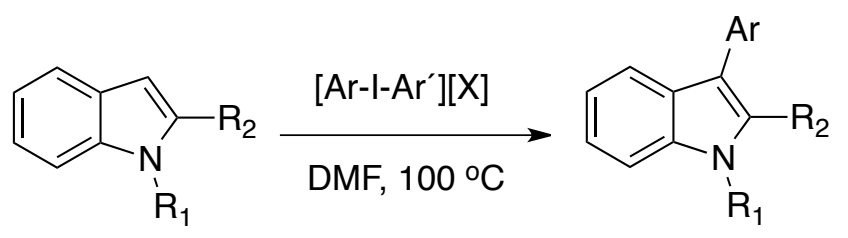

Iodine-Mediated Direct 3-Amination of Indoles:

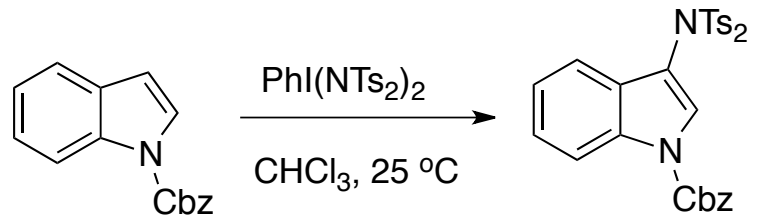

Scheme 1. Iodine(III)-reagents for the selective 3-functionalization of indoles.

We recently became interested in the application of hypervalent iodine(III) reagents such as $\mathrm{PhI}\left(\mathrm{NTs}_{2}\right)_{2}{ }^{7}$ with preformed iodine-nitrogen bonds as uniquely active promoters for the selective amination of hydrocarbon molecules. ${ }^{8,9}$ This chemistry can either be carried out with preformed reagents or through their in situ generation, and greatly surpasses the chemistry of related reagents bearing nitrogen groups such as phthalimide and saccharin, ${ }^{10}$ which require the presence of metal promoters for amination reactions. It has resulted in the development of direct amination reactions such as allylic, ${ }^{11,12}$ acetylenylic, ${ }^{13}$ allenylic, ${ }^{14}$ and aromatic $^{15}$ amination reactions, and the diamination of alkenes ${ }^{16}$ and butadienes. ${ }^{17,18}$ These reagents also enable the position-selective 3-amination of indoles under metal-free conditions (eq. 2). ${ }^{3 \mathrm{a}}$ A related process was reported using NFSI as aminating agent. ${ }^{19}$ Amination of indoles should be considered a particularly interesting topic given the inherent biological diversification that arises from amine incorporation into the aromatic indole core. Consequently, investigation on protocols for additional selective $\mathrm{C}-\mathrm{N}$ bond formation is highly desirable. We herein report our general results on this topic and 
demonstrate that the conceived products can be selectively further diversified into higherfunctionalized molecules.

\section{Results and Discussion}

For indoles with a substituted 3-position, as demonstrated by the introduction of a methyl substituent, the amination reaction with $\mathrm{PhI}\left(\mathrm{NTs}_{2}\right)_{2}$ takes place selectively at the 2-position (Table 1).

Table 1. Selective 2-amination of 3-methyl indoles $\mathbf{1}$ and $\mathbf{2}$.<smiles>Cc1c[nH]c2ccccc12</smiles>

$1(\mathrm{R}=\mathrm{H})$ $2(\mathrm{R}=\mathrm{Me})$

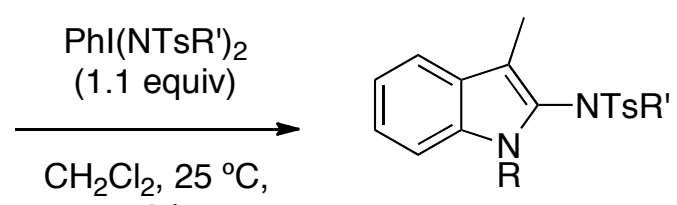

3, 4a,b<smiles></smiles><smiles>Cc1c([N+](=O)[O-])[nH]c2ccccc12</smiles><smiles>Cc1c([N+](C)(C)C)[nH]c2ccccc12</smiles>

$3\left(75 \%, 26 \%{ }^{a}\right)$ 4a $(92 \%)$

4b (98\%)

${ }^{\mathrm{a}}$ Reaction with $\mathrm{PhI}(\mathrm{OAc})_{2} / 2 \mathrm{HNTs}_{2}$

The reaction was explored for free 3-methyl indole $\mathbf{1}$ and its $\mathrm{N}$-methylated derivative $\mathbf{2}$, both of which underwent 2-bistosylimidation to the corresponding products $\mathbf{3}$ and $\mathbf{4 a}$ in good yields. Changing the bissulfonimide group in the hypervalent iodine reagent to mesyltosylimide led to formation of $\mathbf{4 b}$ in excellent yield. As demonstrated for $\mathbf{3}$ as substrate, a reagent combination of (diacetoxy)iodosobenzene and bistosylimide for in situ 
formation of $\mathrm{PhI}\left(\mathrm{NTs}_{2}\right)_{2}$ gives inferior results, which is due to the high reactivity of the indole core. It also appears that N-protected indoles give higher yields, possibly due to nonproductive reactivity of the $\mathrm{N}-\mathrm{H}$ bond in $\mathbf{1}$, while $\mathbf{2}$ reacts with complete selectivity. The successful formation of $\mathbf{3}$ and $\mathbf{4 a , b}$ add to recent work on iodine-mediated 2-amination of indoles ${ }^{19}$ and to a recent non-related 2 -selective amination at the free indole core by Togo, who employed our $\mathrm{PhI}(\mathrm{OAc})_{2} / \mathrm{HNTs}_{2}$ reagent combination. ${ }^{20-22}$

Aiming for a higher functional derivatization, we turned attention to tryptamines as the starting point for structural diversification within amination with hypervalent iodine reagents. In the field of tryptamine alkaloids, the hexahydropyrroloindole derivatives derived from intramolecular 2-amination represent common entities that constitute a fascinating class of natural products. ${ }^{24}$ Tryptamine and derivatives thereof have therefore been of interest for the exploration of oxidative transformations. Interestingly, the parent oxidant (diacetoxy)iodosobenzene is known to enable aminooxygenation of $N$-acetyl tryptamine to form the corresponding 2-acetoxylated fused pyrrolidine products. ${ }^{25}$ In another reaction, we had reported a single example that unlike this reagent, $\mathrm{PhI}\left(\mathrm{NTs}_{2}\right)_{2}$ does not provide the corresponding diamination reaction, but rather provides access to the corresponding 2-aminated tryptamine product. $^{7}$ 
Table 2. Intermolecular 2-amination of tryptamines: scope<smiles>[R7]NCCc1c([NH2+][R5]([H])([H])[H])[nH]c2ccccc12</smiles>

5a-e $(R=H)$

$6 a-i(R=M e)$

7a-e,8a-i<smiles>CCNCCc1c([N+](=O)[O-])[nH]c2ccccc12</smiles>

$7 a(88 \%)$<smiles>O=C(NCCc1c(NC(=O)c2ccccc2)[nH]c2ccccc12)c1ccccc1</smiles>

$7 b(63 \%)$<smiles>[NH3+]c1[nH]c2ccc(OCc3ccccc3)cc2c1CCNC(=O)c1ccccc1</smiles>

7c $(79 \%)$<smiles>CC(C)(C)NCCc1c([N+](=O)[O-])[nH]c2ccccc12</smiles>

$7 d(50 \%)$<smiles>Cn1c([NH3+])c(CCNC(C)(C)C)c2ccccc21</smiles>

$8 a(99 \%)$<smiles>CC(C)NCCc1c([NH3+])n(C)c2ccccc12</smiles>

8d $(87 \%)$<smiles>CNCCc1c(N(C)C)n(C)c2ccccc12</smiles>

$8 g(71 \%)$<smiles>CC(C)=[N+]c1[nH]c2ccccc2c1CCNC(C)C</smiles>

$7 e(62 \%)$

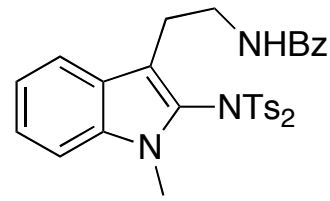

$8 b(92 \%)$<smiles>Cn1c([N-]C(=O)c2ccccc2)c(CCNC(=O)c2ccccc2)c2ccccc21</smiles>

8 e $(90 \%)$<smiles>CN(C)c1c(CCNC(=O)c2ccccc2)c2ccccc2n1C</smiles>

8h $(77 \%)$<smiles>CCCCCC(=O)NCCc1c(N(C)C)n(C)c2ccc(OCc3ccccc3)cc12</smiles>

8c (95\%)<smiles>Cn1c([NH2+]C(=O)c2ccccc2)c(CCNC(C)(C)C)c2cc(OCc3ccccc3)ccc21</smiles>

$8 f(92 \%)$<smiles>CC(C)(C)c1ccccc1</smiles>

$8 \mathbf{i}(80 \%)$

Efficient reactions for intermolecular 2-aminations of tryptamines are apparently rare, since they require overriding the intramolecular cyclization event. We therefore investigated the scope of such a transformation and discovered that the reaction is of general applicability. 
As outlined in Table 2 for 14 examples, differently substituted tryptamines, either free (5ae) or methylated (6a-i) at the aromatic nitrogen, underwent clean 2-amination with complete selectivity in favor of the intermolecular pathway. Transferred nitrogen groups include bistosylimide, bismesylimide and mesyltosylimide alike and the reaction follows the feature from Table 1 that N-protected tryptamines (71-99\% isolated yield) give higher yields that the one with free N-H group (50-88\% isolated yield).

The formed 2-aminated tryptamine derivatives should represent interesting starting points for additional amination/cyclization under electrophilic conditions. ${ }^{24}$ Such a process appeared of interest and was briefly addressed experimentally. At the outset, electrophilic iodonium generated from established $\mathrm{IPy}_{2} \mathrm{BF}_{4}{ }^{26}$ was investigated. Despite experimental variation, the desired iodoamination was never accomplished. Obviously, the size of the bissulfonylimido group at the 2-position of the substrate exercises significant steric shielding of both faces of the $\mathrm{C} 2-\mathrm{C} 3$-double bond of the indole thus preventing electrophilic activation, even with an iodonium of enhanced electrophilicity. Instead, position-selective iodination of the arene core was observed as demonstrated for the reactions of 7a and 8a yielding $\mathbf{9}$ and $\mathbf{1 0}$ in very good to quantitative yield, respectively (Scheme 2). ${ }^{27}$ Forcing the reaction with higher amounts of reagent, additional arene iodination took place at the 6- and the 7-position of 7a, respectively. ${ }^{27}$ Particularly the latter is an interesting outcome, since functionalization at the 7-position of the indole is usually complicated. ${ }^{28}$ Other common iodonium precursors such as NIS or hypervalent iodine reagents such as bis(trifluoroacetoxy)iodosobenzene ${ }^{10 a, b}$ led to no conversion. 
<smiles>CCCCCNc1[nH]c2ccccc2c1CCNC(C)(C)C</smiles>

$3 a$

$4 a$<smiles>CC(C)(C)NCCc1c([N+](C)(C)C)[nH]c2ccccc12</smiles>

$3 a$

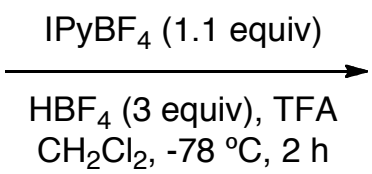

$\mathrm{CH}_{2} \mathrm{Cl}_{2},-78^{\circ} \mathrm{C}, 2 \mathrm{~h}$<smiles>CC(C)(C)NCCc1c([N+](C)(C)C)[nH]c2ccc(I)cc12</smiles>

$9(\mathrm{R}=\mathrm{H}, 87 \%)$

$10(R=M e, 98 \%)$

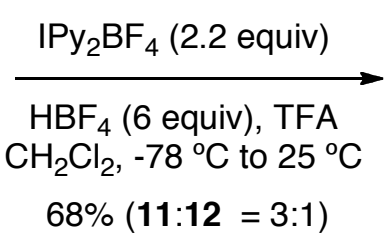<smiles>CC(C)(C)NCCc1c([NH3+])[nH]c2c(I)cc(I)cc12</smiles>

11

Scheme 2. Iodination of 2-aminated tryptamines 7a, 8a.

In order to investigate a smaller electrophile, attention was turned to electrophilic fluorination. To this end, a recent report from Gouvernor on fluoroamination of tryptamines using Selectfluor set the basis to investigate a possible cyclization. ${ }^{29 a}$ Although the reported requirement for cinchona alkaloids shut down any reactivity for the present cases, exposing compounds $\mathbf{7 a , b}$ and $\mathbf{8 a , b}$, respectively, to the presence of Selectfluor and base, resulted in clean fluorination. ${ }^{29}$ However, despite several attempts no accompanying cyclization could be accomplished, but rather detosylation to result in an imino group at $\mathrm{C} 2$ to produce the so far unknown amidine products 13a,b and 14a,b (Scheme 3). Under these conditions, products 13a,b and 14a,b were obtained in almost quantitative yields. These transformations were considered to involve an initial participation of the lone pair of the nitrogen of the indole core. This assumption could be confirmed when the reaction of $7 \mathbf{a}$ was conducted for a shorter period of time, which led to the isolation of compound $\mathbf{1 5}$ as 
the major product in $86 \%$ yield. Exposure of $\mathbf{1 5}$ to an additional period of heating in acetonitrile led to unprecedented detosylation and formation of $\mathbf{1 3 a}$ as the only product.<smiles>O=C(O)NCCc1c([N+](=O)[O-])[nH]c2ccccc12</smiles>

$7 a, b$

$8 a, b$ [7a]<smiles>CC(=O)NCCC1(F)C([N+](=O)[O-])=Nc2ccccc21</smiles>

$15(86 \%)$

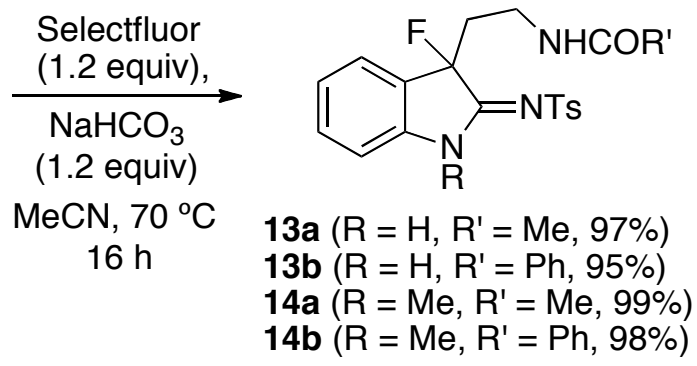

$\left[\right.$ 14a,b] $\mid \begin{array}{l}\mathrm{LiOH}(10 \text { equiv), } \\ \mathrm{MeOH}, \\ 25^{\circ} \mathrm{C}, 12 \mathrm{~h}\end{array}$

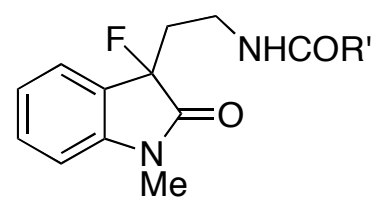

$16 \mathbf{a}\left(R^{\prime}=\mathrm{Me}, 85 \%\right)$ $16 b\left(R^{\prime}=P h, 85 \%\right)$

Scheme 3. Chemical transformation of compounds 7a,b and $\mathbf{8 a}, \mathbf{b}$ upon electrophilic fluorination.

The treatment of $\mathbf{1 4 a , b}$ with lithium hydroxide in methanol provided conditions for a clean hydrolysis of their tosylimido groups leading to their conversion into the corresponding new oxindole derivatives 16a,b. Again, this transformation is of complete selectivity and the two compounds are isolated as the only products in $85 \%$ yield each. When fluorinated compounds 13a,b and 14a,b were exposed to acidic conditions with neat trifluoroacetic acid (TFA), clean defluorination was observed (Scheme 4). 
<smiles>[N+]=[N+]=C1Nc2ccccc2C1(F)CCNC=O</smiles>

13a,b

$14 a, b$<smiles></smiles>
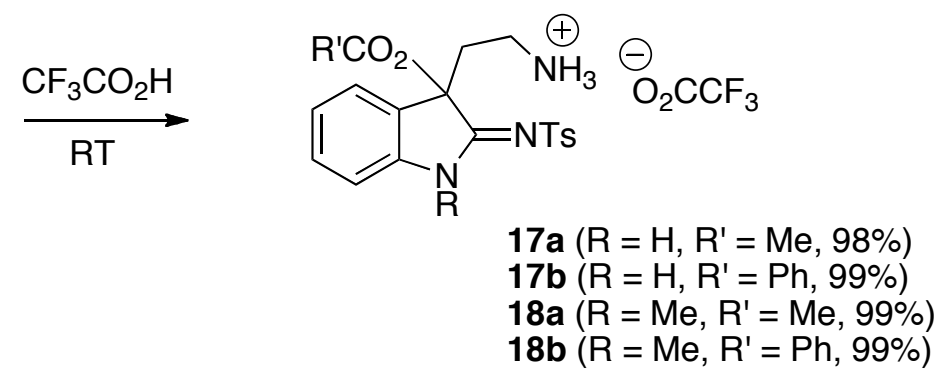

Scheme 4. Chemical transformation of compounds 13a,b and 14a,b.

These conditions led to nucleophilic substitution by the $N$-acetyl and $N$-benzoyl groups from the ethylenylamine side chain at the benzylic position. Upon exposure to moisture during crystallization, they converted in quantitative yields into the benzylic esters $\mathbf{1 7 a}, \mathbf{b}$ and 18a,b, respectively, bearing an ammonium trifluoroacetate group in the side chain. ${ }^{16}$ This overall transformation initiates from $\mathbf{A}$ upon activation of the benzylic fluoride in the presence of TFA as a strong acid, thereby greatly enhancing its leaving group quality. Subsequently, intermediate B forms via intramolecular nucleophilic substitution followed by reversible addition of water to $\mathbf{C}$, from which an intramolecular proton shift afford the products. Note that in the presence of basic conditions, compounds $\mathbf{1 3 a}, \mathbf{b}$ and $\mathbf{1 4 a}, \mathbf{b}$ retain the benzylic fluoride moiety. Exposure of products $\mathbf{1 7 a}, \mathbf{b}$ and $\mathbf{1 8 a}, \mathbf{b}$ to basic conditions 
affords additional diversification (Scheme 5). Treatment with triethylamine generates a free primary amine D from 17a, which initiates quantitative intramolecular acetyl transfer via the tetrahedral intermediate $\mathbf{E}$ to reestablish the acetamide side chain and provides a benzylic hydroxyl group in $\mathbf{1 9} .^{27}$

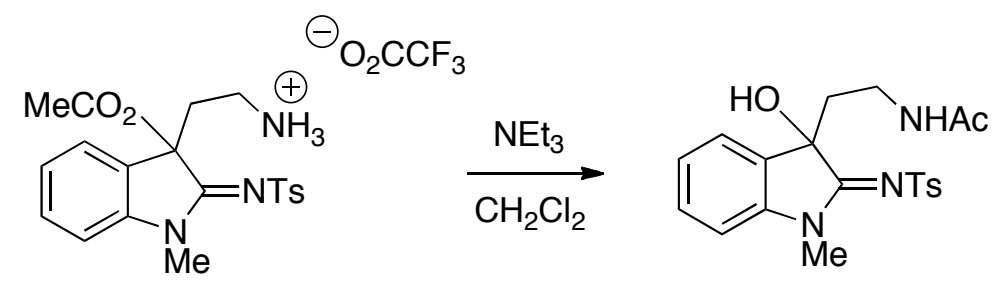

$18 a$

19

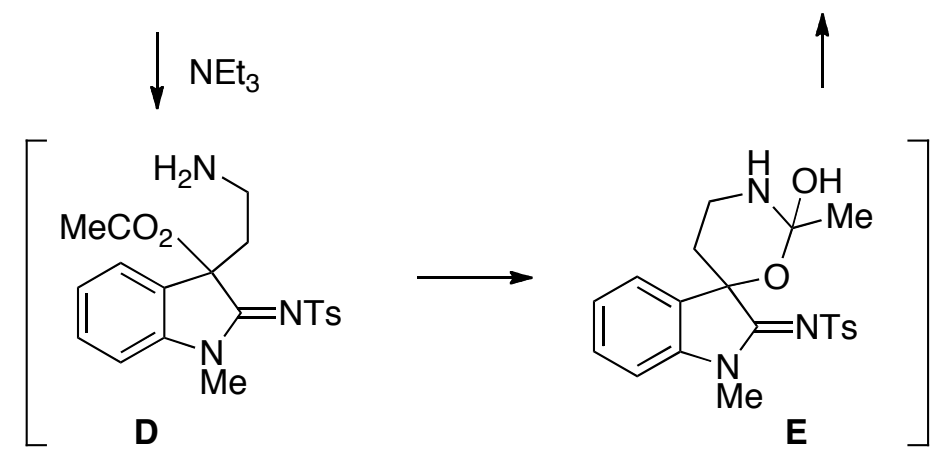

Scheme 5. Chemical transformation of compound 18a to 19.

In contrast, treatment with stronger $\mathrm{LiOH}$ base promotes the expected oxindole formation together with the installment of a spiro-dihydrooxazine functionality in $\mathbf{2 0}{ }^{27}$ Again, this multiple transformation starts from liberation of the free primary amine $\mathbf{F}$ and proceeds through the corresponding tetrahedral intermediate $\mathbf{G}$ with complete selectivity and in quantitative isolated yield (Scheme 6). 
<smiles>CN1C(=[N+]=[N-])C(CC[NH3+])(CC[NH3+])c2ccccc21</smiles>

$18 b$
$\Theta_{\mathrm{O}_{2} \mathrm{CCF}_{3}} \mathrm{LiOH}$
$\stackrel{(10 \text { equiv })}{\mathrm{MeOH}}$

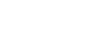<smiles>CN1C(=O)C2(CCN=C(c3ccccc3)O2)c2ccccc21</smiles>

20 (99\%)<smiles>Cc1ccc(C(=O)NCCC2(OC(=O)c3ccccc3)C(=O)Nc3ccccc32)cc1</smiles>

21 (99\%)

$\begin{aligned} & \Theta \mathrm{OH} \\ & \mathrm{H}_{2} \mathrm{O}\end{aligned}-\mathrm{H}_{2} \mathrm{NTS}$<smiles>O=CC(O)C=O</smiles><smiles>CN1C(=O)C(CCN)(OCc2ccccc2)c2ccccc21</smiles><smiles>CN1C(=O)C2(CCNC(O)(c3ccccc3)O2)c2ccccc21</smiles>

Scheme 6. Chemical transformation of compound $\mathbf{1 8 b}$ to 21.

In the presence of TFA, compound $\mathbf{2 0}$ undergoes quantitative opening of the spiro-cyclic arrangement upon concomitant addition of one molecule of TFA to furnish 21 with its benzoylamide side chain and a benzylic trifluoroacetate ester. Exposure to LiOH reverts 21 back to $\mathbf{2 0}$ in essentially quantitative manner. These transformations provide access to a unique number of new tryptamine derivatives. In view of the general occurrence of the 3(aminoethyl)-3-hydroxyoxindole motif (Figure 1), ${ }^{30}$ our new methodology provides unique access to derivatives of this core, and to the additional, yet biologically unexplored classes of 2-imido and allosteric 3-fluoro ${ }^{30}$ derivatives. 
<smiles>CNCCC1(O)C(=O)Nc2ccccc21</smiles>

donoxaridine

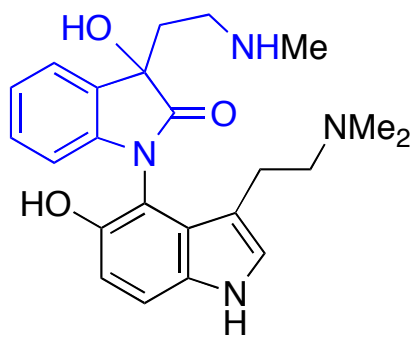

arundaphine

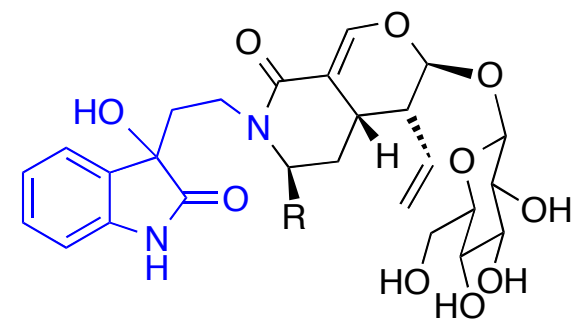

paratunamides

Figure 1. Examples for naturally occurring 3-(aminoethyl)-3-hydroxyoxindoles.

In summary, we have developed a general strategy to intermolecular C2-amination of indoles and tryptamines. This transformation proceeds selectively in the presence of hypervalent iodine reagents of the general formula $\mathrm{PhI}\left[\mathrm{N}\left(\mathrm{SO}_{2} \mathrm{R}\right)\left(\mathrm{SO}_{2} \mathrm{R}^{\prime}\right)\right]$. The corresponding products undergo oxidative fluorination under parallel desulfonylation to provide a new class of tryptamine derivatives. These compounds initiate a number of remarkable transformations, with and without interplay by the ethylenamine side chain. As a result, a higher degree of structural variation is now available for tryptamine derivatives providing building blocks for future exploration.

\section{Experimental Section}

\section{General Remarks}

Chemicals and solvents for chromatography were used as received. Solvents were obtained from a solvent purification system. Reactions that were monitored by TLC were visualized by a dual short wave/long wave UV lamp. ${ }^{1} \mathrm{H},{ }^{13} \mathrm{C}$ and ${ }^{19} \mathrm{~F}$ NMR spectra were recorded using an internal deuterium lock on a 300 or a $500 \mathrm{MHz}$ spectrometer. All chemical shifts 
in NMR experiments are reported as ppm downfield from TMS. $J$ couplings are reported in hertz. The following calibrations were used: $\mathrm{CDCl}_{3} \delta=7.26$ and $77.0 \mathrm{ppm}, \mathrm{CD}_{2} \mathrm{Cl}_{2} \delta=$ 5.32 and $54.00 \mathrm{ppm}, \mathrm{MeOD} \delta=3.31$ and $49.0 \mathrm{ppm}$. Infrared spectra were taken in the solid state and were recorded on a FT-IR fitted with an ATR accessory. Absorptions are given in wavenumbers $\left(\mathrm{cm}^{-1}\right)$. High resolution mass spectra were obtained on a HRMS-TOF spectrometer.

The following compounds were purchased form commercial suppliers and used as received: tryptamine, 5-benzyloxytryptamine, tosyl chloride, mesyl chloride, triethylamine, acetyl chloride, benzoyl chloride, methyl iodide, trifluoroacetic acid, lithium hydroxide, di(acetoxy)iodobenzene, bis(pyridine)iodonium tetrafluoroborate, tetrafluoroboric acid diethyletherate, Selectfluor ${ }^{\circledR}$ and 3-methylindole (1). The following compounds were synthesized according to literature procedures: $2,{ }^{32} \mathbf{5 a},{ }^{33} \mathbf{5 b},{ }^{7} \mathbf{5 c},{ }^{34} \mathbf{5 d},{ }^{35} \mathbf{6 a},{ }^{33}$ and $\mathbf{6 b} .{ }^{33}$

$N$-(2-(5-(Benzyloxy)-1-methyl-1H-indol-3-yl)ethyl)benzamide 6c. A mixture of the indole $5 \mathbf{c}^{33}$ (0.18 g, $0.5 \mathrm{mmol}, 1.0$ equiv), $\mathrm{NaOH}\left(0.06 \mathrm{~g}, 1.5 \mathrm{mmol}, 3\right.$ equiv) and $\mathrm{Bu}_{4} \mathrm{NOH}$ (8 mg, $0.025 \mathrm{mmol}, 5 \% \mathrm{~mol})$ in $\mathrm{CH}_{2} \mathrm{Cl}_{2}(5 \mathrm{~mL})$, was stirred at $25{ }^{\circ} \mathrm{C}$ for $10 \mathrm{~min}$. MeI $(0.08$ $\mathrm{g}, 0.55 \mathrm{mmol}, 1.1$ equiv) was added into the reaction mixture at $25^{\circ} \mathrm{C}$. The reaction mixture was stirred for $12 \mathrm{~h}$. The reaction mixture was quenched with aqueous solution of $10 \% \mathrm{HCl}$ and then with aqueous saturated solution of $\mathrm{NaHCO}_{3}$ and was extracted with $\mathrm{CH}_{2} \mathrm{Cl}_{2}$. The combined organic layers were dried over $\mathrm{Na}_{2} \mathrm{SO}_{4}$. The solvent was removed under reduced pressure. The residue was purified by column chromatography (silica gel, $\mathrm{MeOH} / \mathrm{CH}_{2} \mathrm{Cl}_{2}$, $0.2 / 10, \mathrm{v} / \mathrm{v})$ to afford the pure product $\left(0.096 \mathrm{~g}, 50 \%\right.$ yield, colorless oil). ${ }^{1} \mathrm{H}$ NMR (400 $\left.\mathrm{MHz}, \mathrm{CDCl}_{3}\right): \delta=2.95(\mathrm{t}, J=6.6 \mathrm{~Hz}, 2 \mathrm{H}), 3.63-3.70(\mathrm{~m}, 5 \mathrm{H}), 3.79(\mathrm{td}, J=6.8,6.1 \mathrm{~Hz}$, 
2H), $4.91(\mathrm{~s}, 2 \mathrm{H}), 6.22(\mathrm{~s}, 1 \mathrm{H}), 6.80(\mathrm{~s}, 1 \mathrm{H}), 6.88(\mathrm{dd}, J=8.8,2.4 \mathrm{~Hz}, 1 \mathrm{H}), 7.05(\mathrm{~d}, J=2.4$ $\mathrm{Hz}, 1 \mathrm{H}), 7.12(\mathrm{~d}, J=8.8 \mathrm{~Hz}, 1 \mathrm{H}), 7.22-7.36(\mathrm{~m}, 8 \mathrm{H}), 7.58-7.61(\mathrm{~m}, 2 \mathrm{H}) .{ }^{13} \mathrm{C}$ NMR $(101$ $\left.\mathrm{MHz}, \mathrm{CDCl}_{3}\right): \delta=25.3\left(\mathrm{CH}_{2}\right), 32.8\left(\mathrm{CH}_{3}\right), 40.7\left(\mathrm{CH}_{2}\right), 71.0\left(\mathrm{CH}_{2}\right), 102.3,110.2,111.2$, $112.8,126.9,127.5,127.7,127.8,128.1,128.5,128.6,131.3,132.7,134.7,137.6,153.1$, $167.4(\mathrm{C}=\mathrm{O}) . \quad \mathrm{IR} v\left(\mathrm{~cm}^{-1}\right): 3316(\mathrm{~N}-\mathrm{H}), 3029(\mathrm{Ar}-\mathrm{H}), 2926(\mathrm{Ar}-\mathrm{H}), 2860(\mathrm{Ar}-\mathrm{H}), 1625$ $(\mathrm{C}=\mathrm{O}), 1542,1488,1462,1317,1218,1198,1021,795,695$. HRMS (ESI-TOF): calcd. for $\mathrm{C}_{25} \mathrm{H}_{25} \mathrm{~N}_{2} \mathrm{O}_{2}$ : 385.1911 ; found: 385.1914 .

General procedure for amination reaction indoles and tryptamines. To a solution of the corresponding starting material $\left(0.2 \mathrm{mmol}, 1.0\right.$ equiv) in $\mathrm{CH}_{2} \mathrm{Cl}_{2}(2.0 \mathrm{~mL})$ was added the corresponding iodine(III) reagent $(0.22 \mathrm{mmol}, 1.1$ equiv) and the mixture was stirred at 25 ${ }^{\circ} \mathrm{C}$ for $12 \mathrm{~h}$. After that time, the solvent was removed under reduced pressure and the crude mixture was purified by column chromatography (silica gel, $\mathrm{MeOH} / \mathrm{CH}_{2} \mathrm{Cl}_{2}, 0.2 / 10, \mathrm{v} / \mathrm{v}$ ) to obtain the pure product.

4-Ethyl- $\boldsymbol{N}$-(3-methyl-1 $\boldsymbol{H}$-indol-2-yl)- $\boldsymbol{N}$-tosylbenzenesulfonamide $3.70 \mathrm{mg}, 75 \%$ yield, white solid. m.p. $=164-165{ }^{\circ} \mathrm{C} .{ }^{1} \mathrm{H}$ NMR $\left(400 \mathrm{MHz}, \mathrm{CDCl}_{3}\right): \delta=1.69(\mathrm{~s}, 3 \mathrm{H}), 2.48(\mathrm{~s}, 6 \mathrm{H})$, $7.14(\mathrm{~m}, 1 \mathrm{H}), 7.28-7.31(\mathrm{~m}, 2 \mathrm{H}), 7.34(\mathrm{~d}, J=7.7 \mathrm{~Hz}, 4 \mathrm{H}), 7.51(\mathrm{~d}, J=8.0 \mathrm{~Hz}, 1 \mathrm{H}), 7.82$ (brs, $1 \mathrm{H}), 7.86(\mathrm{~d}, J=8.4 \mathrm{~Hz}, 4 \mathrm{H}) .{ }^{13} \mathrm{C} \mathrm{NMR}\left(125 \mathrm{MHz}, \mathrm{CDCl}_{3}\right): \delta=8.0\left(\mathrm{CH}_{3}\right), 21.8$ $\left(\mathrm{CH}_{3}\right), 111.4,115.6,119.9,120.0,122.7,124.1,127.5,128.6,129.7,135.0,136.4,145.3$. IR $v\left(\mathrm{~cm}^{-1}\right): 3385(\mathrm{~N}-\mathrm{H}), 2956(\mathrm{Ar}-\mathrm{H}), 2923(\mathrm{Ar}-\mathrm{H}), 2851$ (Ar-H), 1595, 1372, 1345, 1169, 1082, 881, 657, 549. HRMS (ESI-TOF): calcd. for $\mathrm{C}_{23} \mathrm{H}_{21} \mathrm{~N}_{2} \mathrm{O}_{4} \mathrm{~S}_{2}$ : 453.0943; found: 453.0927. 
$\boldsymbol{N}$-(1,3-Dimethyl-1H-indol-2-yl)-4-methyl- $\boldsymbol{N}$-tosylbenzenesulfonamide 4 a. $85 \mathrm{mg}, 92 \%$ yield, brown solid. m.p. $=194-196{ }^{\circ} \mathrm{C} .{ }^{1} \mathrm{H}$ NMR $\left(400 \mathrm{MHz}, \mathrm{CDCl}_{3}\right): \delta=1.66(\mathrm{~s}, 3 \mathrm{H}), 2.40$ (s, 6H), 3.19 (s, 3H), 7.03-7.08 (m, 1H), 7.15-7.23 (m, 2H), $7.26(\mathrm{~d}, J=7.7 \mathrm{~Hz}, 4 \mathrm{H}), 7.46$ $(\mathrm{d}, J=8.0 \mathrm{~Hz}, 1 \mathrm{H}), 7.80(\mathrm{~d}, J=8.4 \mathrm{~Hz}, 4 \mathrm{H}) .{ }^{13} \mathrm{C} \mathrm{NMR}\left(101 \mathrm{MHz}, \mathrm{CDCl}_{3}\right): \delta=8.7\left(\mathrm{CH}_{3}\right)$, $21.8\left(\mathrm{CH}_{3}\right), 29.5\left(\mathrm{CH}_{3}\right), 109.8,113.8,119.4,120.0,123.7,124.6,126.4,129.0,129.7$, 136.0, 136.4, 145.5. IR v( $\left.\mathrm{cm}^{-1}\right): 3055$ (Ar-H), 2922 (Ar-H), 1594, 1469, 1372, 1357, 1166, 1084, 878, 738. HRMS (ESI-TOF): calcd. for $\mathrm{C}_{24} \mathrm{H}_{24} \mathrm{~N}_{2} \mathrm{NaO}_{4} \mathrm{~S}_{2}$ : 491.1070; found 491.1065.

$N$-(1,3-dimethyl-1H-indol-2-yl)-4-methyl- $N$-(methylsulfonyl)benzenesulfonamide $\quad 4 b$. $76 \mathrm{mg}, 98 \%$ yield, yellow solid. m.p. $=189-191{ }^{\circ} \mathrm{C} .{ }^{1} \mathrm{H}$ NMR $\left(400 \mathrm{MHz}, \mathrm{CDCl}_{3}\right): \delta=2.01$ (s, 3H), 2.49 (s, 3H), $3.44(\mathrm{~s}, 3 \mathrm{H}), 3.61(\mathrm{~s}, 3 \mathrm{H}), 7.14-7.19(\mathrm{~m}, 1 \mathrm{H}), 7.24-7.32(\mathrm{~m}, 2 \mathrm{H}), 7.35$ $(\mathrm{d}, J=8.0 \mathrm{~Hz}, 2 \mathrm{H}), 7.58(\mathrm{~d}, J=7.9 \mathrm{~Hz}, 1 \mathrm{H}), 7.83(\mathrm{~d}, J=8.4 \mathrm{~Hz}, 2 \mathrm{H}) .{ }^{13} \mathrm{C} \mathrm{NMR}(101 \mathrm{MHz}$, $\left.\mathrm{CDCl}_{3}\right): \delta=8.8\left(\mathrm{CH}_{3}\right), 21.8\left(\mathrm{CH}_{3}\right), 29.4\left(\mathrm{CH}_{3}\right), 44.4\left(\mathrm{CH}_{3}\right), 109.8,113.5,119.5,120.0$ 123.8, 126.3, 129.2, 129.7, 135.1, 135.9, 145.9. IR v( $\left.\mathrm{cm}^{-1}\right): 3055(\mathrm{Ar}-\mathrm{H}), 2931(\mathrm{Ar}-\mathrm{H})$, 1469, 1369, 1354, 1163, 1121, 1087, 966, 872, 737. HRMS (ESI-TOF): calcd. for $\mathrm{C}_{18} \mathrm{H}_{20} \mathrm{~N}_{2} \mathrm{NaO}_{4} \mathrm{~S}_{2}$ : 415.0757; found 415.0743.

N-(2-(2-(4-Methyl-N-tosylphenylsulfonamido)-1H-indol-3-yl)ethyl)acetamide $\quad 7 a . \quad 87$ mg, 83\% yield, white solid. m.p. $=170-171^{\circ} \mathrm{C} .{ }^{1} \mathrm{H}$ NMR $\left(400 \mathrm{MHz}, \mathrm{CDCl}_{3}\right): \delta=1.79(\mathrm{~s}$, $3 \mathrm{H}), 2.16-2.20(\mathrm{t}, J=6.7,2 \mathrm{H}), 2.48(\mathrm{~s}, 6 \mathrm{H}), 3.53(\mathrm{td}, J=6.2,5.8 \mathrm{~Hz}, 2 \mathrm{H}), 6.24$ (brs, $1 \mathrm{H})$, 7.13-7.17 (m, 1H), 7.28-7.35 (m, 2H), $7.36(\mathrm{~d}, J=8.1 \mathrm{~Hz}, 4 \mathrm{H}), 7.62(\mathrm{~d}, J=8.0 \mathrm{~Hz}, 1 \mathrm{H})$, $7.86(\mathrm{~d}, J=8.4 \mathrm{~Hz}, 4 \mathrm{H}), 8.30(\mathrm{~s}, 1 \mathrm{H}) .{ }^{13} \mathrm{C} \mathrm{NMR}\left(101 \mathrm{MHz}, \mathrm{CDCl}_{3}\right): \delta=21.8\left(\mathrm{CH}_{3}\right), 22.9$ 
$\left(\mathrm{CH}_{3}\right), 23.0\left(\mathrm{CH}_{2}\right), 38.6\left(\mathrm{CH}_{2}\right), 111.8,116.2,120.3,120.4,123.4,124.5,126.4,128.7$, 129.9, 135.2, 135.8, 145.9, $170.4(\mathrm{C}=\mathrm{O})$. IR $v\left(\mathrm{~cm}^{-1}\right): 3376(\mathrm{~N}-\mathrm{H}), 3257(\mathrm{~N}-\mathrm{H}), 2922(\mathrm{Ar}-$ H), $1659(\mathrm{C}=\mathrm{O}), 1536,1377,1353,1167,876,773,659,540$. HRMS (ESI-TOF): calcd. for $\mathrm{C}_{26} \mathrm{H}_{27} \mathrm{~N}_{3} \mathrm{O}_{5} \mathrm{NaS}_{2}: 548.1290$, found: 548.1277 .

$N$-(2-(2-(4-Methyl- $N$-tosylphenylsulfonamido)-1H-indol-3-yl)ethyl)benzamide 7 b. $^{7} 74$ mg, 63\% yield, white solid. ${ }^{1} \mathrm{H}$ NMR (400 MHz, $\left.\mathrm{CDCl}_{3}\right): \delta=2.37$ (t, $\left.J=6.7 \mathrm{~Hz}, 2 \mathrm{H}\right), 2.47$ (s, 6H), 3.73 (td, $J=6.7,5.1 \mathrm{~Hz}, 2 \mathrm{H}), 6.67$ (brs, 1H), 7.10-7.15 (m, 1H), 7.28-7.39 (m, 9H),

7.66-7.69 (m, 2H), $7.72(\mathrm{~d}, J=8.0 \mathrm{~Hz}, 1 \mathrm{H}), 7.87(\mathrm{~d}, J=8.4 \mathrm{~Hz}, 4 \mathrm{H}), 8.02$ (brs, $1 \mathrm{H}) .{ }^{13} \mathrm{C}$ NMR (101 MHz, $\left.\mathrm{CDCl}_{3}\right): \delta=21.8\left(\mathrm{CH}_{3}\right), 23.2\left(\mathrm{CH}_{2}\right), 39.3\left(\mathrm{CH}_{2}\right), 111.7,116.3,120.4$, $120.7,123.2,124.5,126.6,127.1,128.2,128.7,129.9,131.1,134.4,135.1,135.9,145.8$ $167.7(\mathrm{C}=\mathrm{O})$.

\section{$N$-(2-(5-(Benzyloxy)-2-(4-methyl- $N$-tosylphenylsulfonamido)-1 H-indol-3-}

yl)ethyl)benzamide 7c. $110 \mathrm{mg}, 80 \%$ yield, brown solid. m.p. $=109-111^{\circ} \mathrm{C} .{ }^{1} \mathrm{H}$ NMR $(400$ $\left.\mathrm{MHz}, \mathrm{CDCl}_{3}\right): \delta=2.33(\mathrm{t}, J=6.6 \mathrm{~Hz}, 2 \mathrm{H}), 2.48(\mathrm{~s}, 6 \mathrm{H}), 3.70(\mathrm{td}, J=6.6,5.2 \mathrm{~Hz}, 2 \mathrm{H}), 4.94$ (s, 2H), 6.65 (t, $J=5.2 \mathrm{~Hz}, 1 \mathrm{H}), 7.00(\mathrm{dd}, J=8.8,2.4 \mathrm{~Hz}, 1 \mathrm{H}), 7.19$ (d, $J=2.4 \mathrm{~Hz}, 1 \mathrm{H})$, $7.23(\mathrm{~d}, J=8.9 \mathrm{~Hz}, 1 \mathrm{H}), 7.30-7.43(\mathrm{~m}, 12 \mathrm{H}), 7.68-7.70(\mathrm{~m}, 2 \mathrm{H}), 7.85-7.88(\mathrm{~m}, 5 \mathrm{H}) .{ }^{13} \mathrm{C}$ NMR (101 MHz, $\left.\mathrm{CDCl}_{3}\right): \delta=21.8\left(\mathrm{CH}_{3}\right), 23.2\left(\mathrm{CH}_{2}\right), 39.2\left(\mathrm{CH}_{2}\right), 70.6\left(\mathrm{CH}_{2}\right)$, 103.0, $112.6,116.0,116.2,123.6,127.1,127.8,127.8,128.3,128.5,128.7,130.0,130.2,130.3$ 131.1, 134.4, 135.9, 137.2, 137.5, 145.8, 153.6, $167.6(\mathrm{C}=\mathrm{O}) . \mathrm{IR} v\left(\mathrm{~cm}^{-1}\right): 3384(\mathrm{~N}-\mathrm{H}), 3191$ (Ar-H), 3059 (Ar-H), 2922 (Ar-H), 1640 (C=O), 1579, 1485, 1376, 1187, 1165, 1083, 882. HRMS (ESI-TOF): calcd. for $\mathrm{C}_{38} \mathrm{H}_{35} \mathrm{~N}_{3} \mathrm{NaO}_{6} \mathrm{~S}_{2}$ : 716.1859; found: 716.1863 . 
tert-Butyl(2-(2-(4-methyl- $N$-tosylphenylsulfonamido)-1H-indol-3-yl)ethyl)carbamate

7d. $58 \mathrm{mg}, 50 \%$ yield, yellow solid. m.p. $=102-107{ }^{\circ} \mathrm{C} .{ }^{1} \mathrm{H} \mathrm{NMR}\left(400 \mathrm{MHz}, \mathrm{CDCl}_{3}\right): \delta=$ $1.42(\mathrm{~s}, 9 \mathrm{H}), 2.26(\mathrm{t}, J=7.2 \mathrm{~Hz}, 2 \mathrm{H}), 2.48(\mathrm{~s}, 6 \mathrm{H}), 3.24-3.29(\mathrm{~m}, 2 \mathrm{H}), 4.73$ (brs, 1H), 7.12$7.16(\mathrm{~m}, 1 \mathrm{H}), 7.28-7.30(\mathrm{~m}, 2 \mathrm{H}), 7.35(\mathrm{~d}, J=7.9 \mathrm{~Hz}, 4 \mathrm{H}), 7.68(\mathrm{~d}, J=7.8 \mathrm{~Hz}, 1 \mathrm{H}), 7.86$ $(\mathrm{d}, J=8.4 \mathrm{~Hz}, 4 \mathrm{H}), 7.99$ (brs, $1 \mathrm{H}) .{ }^{13} \mathrm{C} \mathrm{NMR}\left(101 \mathrm{MHz}, \mathrm{CDCl}_{3}\right): \delta=21.7\left(\mathrm{CH}_{3}\right), 24.2$ $\left(\mathrm{CH}_{2}\right), 28.4\left(\mathrm{CH}_{3}\right), 39.8\left(\mathrm{CH}_{2}\right), 78.8,111.5,116.6,120.1,120.7,122.9,124.2,126.7,128.7$, 129.8, 135.0, 136.0, 145.6, $155.9(\mathrm{C}=\mathrm{O})$. IR $v\left(\mathrm{~cm}^{-1}\right): 3294(\mathrm{~N}-\mathrm{H}), 2927(\mathrm{Ar}-\mathrm{H}), 1632$ $(\mathrm{C}=\mathrm{O}), 1580,1470,1282,1145,1082,770,751,671,545,461$. HRMS (ESI-TOF): calcd. for $\mathrm{C}_{29} \mathrm{H}_{33} \mathrm{~N}_{3} \mathrm{O}_{6} \mathrm{NaS}_{2}: 606.1708$, found: 606.1690 .

\section{$N$-(2-(2-(4-Methyl- $N$-(methylsulfonyl)phenylsulfonamido)-1 $H$-indol-3-}

yl)ethyl)acetamide 7e. $52 \mathrm{mg}, 58 \%$ yield, yellow solid. m.p. $=166-172{ }^{\circ} \mathrm{C} .{ }^{1} \mathrm{H}-\mathrm{NMR}(400$ $\left.\mathrm{MHz}, \mathrm{CDCl}_{3}\right): \delta=1.83(\mathrm{~s}, 3 \mathrm{H}), 2.28-2.36(\mathrm{~m}, 1 \mathrm{H}), 2.48(\mathrm{~s}, 3 \mathrm{H}), 2.58-2.64(\mathrm{~m}, 1 \mathrm{H}), 3.57$ $(\mathrm{td}, J=6.1,5.5 \mathrm{~Hz}, 2 \mathrm{H}), 3.64(\mathrm{~s}, 3 \mathrm{H}), 6.02(\mathrm{~s}, 1 \mathrm{H}), 7.15-7.18(\mathrm{~m}, 1 \mathrm{H}), 7.31-7.35(\mathrm{~m}, 4 \mathrm{H})$, $7.64(\mathrm{~d}, J=8.0 \mathrm{~Hz}, 1 \mathrm{H}), 7.77(\mathrm{~d}, J=8.4 \mathrm{~Hz}, 2 \mathrm{H}), 8.10(\mathrm{~s}, 1 \mathrm{H}) .{ }^{13} \mathrm{C}$ NMR $(101 \mathrm{MHz}$ $\left.\mathrm{CDCl}_{3}\right): \delta=21.8\left(\mathrm{CH}_{3}\right), 23.0\left(\mathrm{CH}_{2}\right), 23.3\left(\mathrm{CH}_{3}\right), 38.6\left(\mathrm{CH}_{2}\right), 44.2\left(\mathrm{CH}_{3}\right), 111.8,115.3$, $120.2,120.3122 .8,124.4,126.2,128.9,129.9,134.6,135.1,146.2,170.7(\mathrm{C}=\mathrm{O}) . \mathrm{IR} v\left(\mathrm{~cm}^{-}\right.$ 1): $3358(\mathrm{~N}-\mathrm{H}), 2928(\mathrm{Ar}-\mathrm{H}), 2880(\mathrm{Ar}-\mathrm{H}), 1646(\mathrm{C}=\mathrm{O}), 1550,1369,1347,1165,976,877$, 744, 664, 520. HRMS (ESI-TOF): calcd. for $\mathrm{C}_{20} \mathrm{H}_{23} \mathrm{~N}_{3} \mathrm{O}_{5} \mathrm{NaS}_{2}: 472.0966$, found: 472.0977 .

\section{$N$-(2-(1-Methyl-2-((4-methyl- $N$-tosylphenyl)sulfonamido)-1H-indol-3-}

yl)ethyl)acetamide 8a. $107 \mathrm{mg}$, 99\% yield, yellow solid. m.p. = 189-190 ${ }^{\circ} \mathrm{C} .{ }^{1} \mathrm{H}$ NMR (400 
$\left.\mathrm{MHz}, \mathrm{CDCl}_{3}\right): \delta=1.82(\mathrm{~s}, 3 \mathrm{H}), 2.25(\mathrm{t}, J=6.5 \mathrm{~Hz}, 2 \mathrm{H}), 2.53(\mathrm{~s}, 6 \mathrm{H}), 3.36(\mathrm{~s}, 3 \mathrm{H}), 3.57-$ $3.61(\mathrm{~m}, 2 \mathrm{H}), 6.20(\mathrm{brs}, 1 \mathrm{H}), 7.17-7.20(\mathrm{~m}, 1 \mathrm{H}), 7.32(\mathrm{~d}, J=8.3 \mathrm{~Hz}, 1 \mathrm{H}), 7.36-7.39(\mathrm{~m}$, $1 \mathrm{H}), 7.40(\mathrm{~d}, J=7.8 \mathrm{~Hz}, 4 \mathrm{H}), 7.70(\mathrm{~d}, J=8.1 \mathrm{~Hz}, 1 \mathrm{H}), 7.90(\mathrm{~d}, J=8.4 \mathrm{~Hz}, 4 \mathrm{H}) .{ }^{13} \mathrm{C} \mathrm{NMR}$ $\left(101 \mathrm{MHz}, \mathrm{CDCl}_{3}\right): \delta=21.8\left(\mathrm{CH}_{3}\right), 23.0\left(\mathrm{CH}_{3}\right), 23.7\left(\mathrm{CH}_{2}\right), 30.0\left(\mathrm{CH}_{3}\right), 38.8\left(\mathrm{CH}_{2}\right), 110.2$, $114.7,120.0,120.6,124.1,125.1,125.5,129.2,129.8,135.8,136.3,146.0,170.3(\mathrm{C}=\mathrm{O})$. IR $v\left(\mathrm{~cm}^{-1}\right): 3399(\mathrm{~N}-\mathrm{H}), 3059(\mathrm{Ar}-\mathrm{H}), 2928(\mathrm{Ar}-\mathrm{H}), 1652(\mathrm{C}=\mathrm{O})$, 1595, 1534, 1471, 1431, 1373, 1358, 1166, 1083, 1017. HRMS (ESI-TOF): calcd. for $\mathrm{C}_{27} \mathrm{H}_{30} \mathrm{~N}_{3} \mathrm{O}_{5} \mathrm{~S}_{2}: 540.1621$; found: 540.1630 .

\section{$N$-(2-(1-Methyl-2-(4-methyl- $N$-tosylphenyl)sulfonamido)-1 $H$-indol-3-}

yl)ethyl)benzamide 8b. $110 \mathrm{mg}, 92 \%$ yield, yellow solid. m.p. $=178-180{ }^{\circ} \mathrm{C} .{ }^{1} \mathrm{H}$ NMR $\left(400 \mathrm{MHz}, \mathrm{CDCl}_{3}\right): \delta=2.46(\mathrm{t}, J=6.9 \mathrm{~Hz}, 2 \mathrm{H}), 2.51(\mathrm{~s}, 6 \mathrm{H}), 3.28(\mathrm{~s}, 3 \mathrm{H}), 3.83(\mathrm{td}, J=6.9$, $5.1 \mathrm{~Hz}, 2 \mathrm{H}), 6.70(\mathrm{t}, J=5.0 \mathrm{~Hz}, 1 \mathrm{H}), 7.15-7.18(\mathrm{~m}, 1 \mathrm{H}), 7.28-7.30(\mathrm{~m}, 1 \mathrm{H}), 7.33-7.36(\mathrm{~m}$, 3H), 7.39-7.44 (m, 5H), 7.70-7.73 (m, 2H), $7.81(\mathrm{~d}, J=8.1 \mathrm{~Hz}, 1 \mathrm{H}), 7.92(\mathrm{~d}, J=8.4 \mathrm{~Hz}$,

4H). ${ }^{13} \mathrm{C} \mathrm{NMR}\left(101 \mathrm{MHz}, \mathrm{CDCl}_{3}\right): \delta=21.8\left(\mathrm{CH}_{3}\right), 23.9\left(\mathrm{CH}_{2}\right), 29.8\left(\mathrm{CH}_{3}\right), 39.5\left(\mathrm{CH}_{2}\right)$, $110.1,114.6,120.0,120.9,124.0,125.1,125.6,127.0,128.2,129.1,129.8,131.0,134.5$ 135.9, 136.2, 145.9, $167.6(\mathrm{C}=\mathrm{O})$. IR $v\left(\mathrm{~cm}^{-1}\right): 3242(\mathrm{~N}-\mathrm{H}), 3069(\mathrm{Ar}-\mathrm{H}), 2923(\mathrm{Ar}-\mathrm{H})$, $1630(\mathrm{C}=\mathrm{O}), 1549,1492,1470,1386,1357,1334,1169,1084,1016$. HRMS (ESI-TOF): calcd. for $\mathrm{C}_{33} \mathrm{H}_{27} \mathrm{~N}_{7} \mathrm{NaOS}_{2}$ : 624.1611; found: 624.1612 .

\section{$N$-(2-(5-(Benzyloxy)-1-methyl-2-(4-methyl- $N$-tosylphenylsulfonamido)-1H-indol-3-}

yl)ethyl)benzamide 8c. $133 \mathrm{mg}, 95 \%$ yield, orange solid. m.p. $=168-170{ }^{\circ} \mathrm{C} .{ }^{1} \mathrm{H}$ NMR $\left(400 \mathrm{MHz}, \mathrm{CDCl}_{3}\right): \delta=2.41(\mathrm{t}, J=6.7 \mathrm{~Hz}, 2 \mathrm{H}), 2.51(\mathrm{~s}, 6 \mathrm{H}), 3.26(\mathrm{~s}, 3 \mathrm{H}), 3.80(\mathrm{td}, J=6.8$, 
$5.2 \mathrm{~Hz}, 2 \mathrm{H}), 5.02(\mathrm{~s}, 2 \mathrm{H}), 6.68(\mathrm{t}, J=5.2 \mathrm{~Hz}, 1 \mathrm{H}), 7.08(\mathrm{dd}, J=8.9,2.3 \mathrm{~Hz}, 1 \mathrm{H}), 7.20(\mathrm{~d}, J$ $=8.9 \mathrm{~Hz}, 1 \mathrm{H}), 7.28(\mathrm{~d}, J=2.1 \mathrm{~Hz}, 1 \mathrm{H}), 7.33-7.42(\mathrm{~m}, 10 \mathrm{H}), 7.46-7.48(\mathrm{~m}, 2 \mathrm{H}), 7.71-7.73$ (m, 2H), 7.91-7.93 (m, 4H). ${ }^{13} \mathrm{C}$ NMR (101 MHz, $\left.\mathrm{CDCl}_{3}\right): \delta=21.8\left(\mathrm{CH}_{3}\right), 23.9\left(\mathrm{CH}_{2}\right), 29.9$ $\left(\mathrm{CH}_{3}\right), 39.4\left(\mathrm{CH}_{2}\right), 70.7\left(\mathrm{CH}_{2}\right), 103.1,111.1,114.2,125.2,125.8,127.0,127.1,127.7$, $127.8,128.3,128.4,128.5,129.1,129.8,129.9,131.1,131.6,134.5,135.9,137.3,145.9$, 153.4, $167.6(\mathrm{C}=\mathrm{O})$. IR $v\left(\mathrm{~cm}^{-1}\right): 3395(\mathrm{~N}-\mathrm{H}), 3032(\mathrm{Ar}-\mathrm{H}), 2924(\mathrm{Ar}-\mathrm{H}), 1645(\mathrm{C}=\mathrm{O})$, 1596, 1487, 1374, 1166, 1083, 1019, 872. HRMS (ESI-TOF): calcd. for $\mathrm{C}_{39} \mathrm{H}_{37} \mathrm{~N}_{3} \mathrm{NaO}_{6} \mathrm{~S}_{2}$ : 730.2016; found: 730.2048 .

\section{$N$-(2-(1-Methyl-2-((4-methyl- $N$-(methylsulfonyl)phenyl)sulfonamido)-1H-indol-3-}

yl)ethyl)acetamide 8d. $80 \mathrm{mg}, 87 \%$ yield, yellow solid. m.p. $=119-121{ }^{\circ} \mathrm{C} .{ }^{1} \mathrm{H}$ NMR $(400$ $\left.\mathrm{MHz}, \mathrm{CDCl}_{3}\right): \delta=1.84(\mathrm{~s}, 3 \mathrm{H}), 2.39-2.44(\mathrm{~m}, 1 \mathrm{H}), 2.50(\mathrm{~s}, 3 \mathrm{H}), 2.68-2.73(\mathrm{~m}, 1 \mathrm{H}), 3.39(\mathrm{~s}$, 3H), 3.54-3.60 (m, 1H), 3.62-3.68 (m, 1H), $3.73(\mathrm{~s}, 3 \mathrm{H}), 5.95(\mathrm{~s}, 1 \mathrm{H}), 7.17-7.20(\mathrm{~m}, 1 \mathrm{H})$, $7.31(\mathrm{~d}, J=8.3 \mathrm{~Hz}, 1 \mathrm{H}), 7.35-7.37(\mathrm{~m}, 3 \mathrm{H}), 7.68(\mathrm{~d}, J=8.1 \mathrm{~Hz}, 1 \mathrm{H}), 7.78(\mathrm{~d}, J=8.4 \mathrm{~Hz}$, 2H). ${ }^{13} \mathrm{C}$ NMR (101 MHz, $\left.\mathrm{CDCl}_{3}\right): \delta=21.8\left(\mathrm{CH}_{3}\right), 23.0\left(\mathrm{CH}_{3}\right), 24.1\left(\mathrm{CH}_{2}\right), 29.5\left(\mathrm{CH}_{3}\right)$, $38.9\left(\mathrm{CH}_{2}\right), 44.6\left(\mathrm{CH}_{3}\right), 110.2,114.4,120.1,120.4,124.2,124.6,125.3,129.2,129.9$, 134.5, 136.2, 146.3, $170.4(\mathrm{C}=\mathrm{O})$. IR $v\left(\mathrm{~cm}^{-1}\right): 3316(\mathrm{~N}-\mathrm{H}), 3017(\mathrm{Ar}-\mathrm{H}), 2982(\mathrm{Ar}-\mathrm{H})$, $2936(\mathrm{Ar}-\mathrm{H}), 1642(\mathrm{C}=\mathrm{O}), 1549,1448,1368,1355,1345,1295,1167,1087$. HRMS (ESITOF): calcd. for $\mathrm{C}_{21} \mathrm{H}_{25} \mathrm{~N}_{3} \mathrm{NaO}_{5} \mathrm{~S}_{2}$ : 486.1128 ; found: 486.1135 .

\section{$N$-(2-(1-Methyl-2-(4-methyl- $N$-(methylsulfonyl)phenyl)sulfonamido)-1 $H$-indol-3-}

yl)ethyl)benzamide 8e. $94 \mathrm{mg}, 90 \%$ yield, yellow solid. m.p. $=182-184{ }^{\circ} \mathrm{C} .{ }^{1} \mathrm{H}$ NMR $(400$ $\left.\mathrm{MHz}, \mathrm{CDCl}_{3}\right): \delta=2.48(\mathrm{~s}, 3 \mathrm{H}), 2.57-2.67(\mathrm{~m}, 1 \mathrm{H}), 2.81-2.90(\mathrm{~m}, 1 \mathrm{H}), 3.36(\mathrm{~s}, 3 \mathrm{H}), 3.66(\mathrm{~s}$, 
3H), 3.81-3.89 (m, 2H), $6.55(\mathrm{~s}, 1 \mathrm{H})$, 7.14-7.19 (m, 2H), 7.30-7.39 (m, 5H), 7.42-7.45 (m, 1H), 7.68-7.71 (m, 2H), 7.75-7.80 (m, 3H). ${ }^{13} \mathrm{C}$ NMR (101 MHz, $\left.\mathrm{CDCl}_{3}\right): \delta=21.8\left(\mathrm{CH}_{3}\right)$, $24.4\left(\mathrm{CH}_{2}\right), 29.4\left(\mathrm{CH}_{3}\right), 39.6\left(\mathrm{CH}_{2}\right), 44.5\left(\mathrm{CH}_{3}\right), 110.1,114.4,120.1,120.6,124.1,124.5$, 125.4, 127.1, 128.3, 129.2, 129.9, 131.2, 134.4, 134.6, 136.2, 146.3, $167.7(\mathrm{C}=\mathrm{O}) . \mathrm{IR} v\left(\mathrm{~cm}^{-}\right.$

1): $3300(\mathrm{~N}-\mathrm{H}), 3043(\mathrm{Ar}-\mathrm{H}), 2929(\mathrm{Ar}-\mathrm{H}), 1636(\mathrm{C}=\mathrm{O}), 1598,1579,1538,1488,1470$, 1429, 1356, 1309, 1165, 1086. HRMS (ESI-TOF): calcd. for $\mathrm{C}_{26} \mathrm{H}_{27} \mathrm{~N}_{3} \mathrm{NaO}_{5} \mathrm{~S}_{2}: 548.1284$; found: 548.1291 .

$N$-(2-(5-(Benzyloxy)-1-methyl-2-(4-methyl- $N$-(methylsulfonyl)phenyl sulfonamido)$\mathbf{1 H}$-indol-3-yl)ethyl)benzamide 8f. $115 \mathrm{mg}, 92 \%$ yield, orange solid. m.p. $=146-148{ }^{\circ} \mathrm{C}$. ${ }^{1} \mathrm{H}$ NMR $\left(400 \mathrm{MHz}, \mathrm{CDCl}_{3}\right): \delta=2.49(\mathrm{~s}, 3 \mathrm{H}), 2.52-2.62(\mathrm{~m}, 1 \mathrm{H}), 2.77-2.87(\mathrm{~m}, 1 \mathrm{H}), 3.34$ (s, 3H), $3.66(\mathrm{~s}, 3 \mathrm{H}), 3.83(\mathrm{td}, J=6.6,6.0 \mathrm{~Hz}, 2 \mathrm{H}), 5.04(\mathrm{q}, J=11.4 \mathrm{~Hz}, 2 \mathrm{H}), 6.53(\mathrm{t}, J=$ $5.5 \mathrm{~Hz}, 1 \mathrm{H}), 7.07(\mathrm{dd}, J=8.9,2.4 \mathrm{~Hz}, 1 \mathrm{H}), 7.20(\mathrm{~d}, J=8.9 \mathrm{~Hz}, 1 \mathrm{H}), 7.24(\mathrm{~d}, J=2.4 \mathrm{~Hz}$, 1H), 7.34-7.47(m, 10H), 7.69-7.72 (m, 2H), $7.80(\mathrm{~d}, J=8.4 \mathrm{~Hz}, 2 \mathrm{H}) .{ }^{13} \mathrm{C}$ NMR $(101 \mathrm{MHz}$, $\left.\mathrm{CDCl}_{3}\right): \delta=21.8\left(\mathrm{CH}_{3}\right), 24.4\left(\mathrm{CH}_{2}\right), 29.6\left(\mathrm{CH}_{3}\right), 39.6\left(\mathrm{CH}_{2}\right), 44.5\left(\mathrm{CH}_{3}\right), 70.7\left(\mathrm{CH}_{2}\right)$, 103.0, 111.1, 113.9, 115.7, 124.6, 125.6, 127.1, 127.7, 128.4, 128.5, 129.2, 129.9, 131.2, 131.6, 134.4, 134.6, 136.2, 137.2, 146.3, $167.6(\mathrm{C}=\mathrm{O}) . \mathrm{IR} v\left(\mathrm{~cm}^{-1}\right): 3410(\mathrm{~N}-\mathrm{H}), 3033(\mathrm{Ar}-$ H), 2928 (Ar-H), $1648(\mathrm{C}=\mathrm{O})$, 1486, 1370, 1353, 1163, 1086, 1025, 966, 873. HRMS (ESITOF): calcd. for $\mathrm{C}_{33} \mathrm{H}_{33} \mathrm{~N}_{3} \mathrm{NaO}_{6} \mathrm{~S}_{2}: 654.1703$; found: 654.1709 .

\section{$N$-(2-(1-Methyl-2-( $N$-(methylsulfonyl)methylsulfonamido)-1 $H$-indol-3-}

yl)ethyl)acetamide 8g. $54 \mathrm{mg}, 71 \%$ yield, white solid. m.p. $=209-210{ }^{\circ} \mathrm{C} .{ }^{1} \mathrm{H}$ NMR $(400$ $\left.\mathrm{MHz}, \mathrm{CDCl}_{3}\right): \delta=1.90(\mathrm{~s}, 3 \mathrm{H}), 3.06(\mathrm{t}, J=7.1 \mathrm{~Hz}, 2 \mathrm{H}), 3.55(\mathrm{~s}, 6 \mathrm{H}), 3.74-3.78(\mathrm{~m}, 5 \mathrm{H})$, 
$5.99(\mathrm{~s}, 1 \mathrm{H}), 7.19-7.23(\mathrm{~m}, 1 \mathrm{H}), 7.36-7.41(\mathrm{~m}, 2 \mathrm{H}), 7.73(\mathrm{~d}, J=8.1 \mathrm{~Hz}, 1 \mathrm{H}) .{ }^{13} \mathrm{C}$ NMR $(101$

$\left.\mathrm{MHz}, \mathrm{CDCl}_{3}\right): \delta=23.1\left(\mathrm{CH}_{3}\right), 25.1\left(\mathrm{CH}_{2}\right), 29.8\left(\mathrm{CH}_{3}\right), 38.9\left(\mathrm{CH}_{2}\right), 43.3\left(\mathrm{CH}_{3}\right), 110.2$, 114.0, 120.3, 120.4, 124.1, 124.4, 125.3, 136.3, $170.4(\mathrm{C}=\mathrm{O}) . \mathrm{IR} v\left(\mathrm{~cm}^{-1}\right): 3425(\mathrm{~N}-\mathrm{H}), 3023$ (Ar-H), 3002 (Ar-H), 2942 (Ar-H), 2923 (Ar-H), 1663 (C=O), 1531, 1469, 1361, 1347, 1290, 1225, 1158, 1101. HRMS (ESI-TOF): calcd. for $\mathrm{C}_{15} \mathrm{H}_{21} \mathrm{~N}_{3} \mathrm{NaO}_{5} \mathrm{~S}_{2}: 410.0815$; found: 410.0815.

\section{$N$-(2-(1-Methyl-2-( $N$-(methylsulfonyl)methylsulfonamido)-1 $H$-indol-3-}

yl)ethyl)benzamide 8h. $69 \mathrm{mg}, 77 \%$ yield, white solid. m.p. $=166-168{ }^{\circ} \mathrm{C} .{ }^{1} \mathrm{H}$ NMR $(400$ $\left.\mathrm{MHz}, \mathrm{CDCl}_{3}\right): \delta=3.19(\mathrm{t}, J=7.0 \mathrm{~Hz}, 2 \mathrm{H}), 3.53(\mathrm{~s}, 6 \mathrm{H}), 3.76(\mathrm{~s}, 3 \mathrm{H}), 3.99(\mathrm{td}, J=7.0,5.6$ $\mathrm{Hz}, 2 \mathrm{H}), 6.59(\mathrm{~s}, 1 \mathrm{H}), 7.18-7.22(\mathrm{~m}, 1 \mathrm{H}), 7.36-7.42(\mathrm{~m}, 4 \mathrm{H}), 7.46-7.50(\mathrm{~m}, 1 \mathrm{H}), 7.72-7.75$ (m, 2H), $7.82(\mathrm{~d}, J=8.0 \mathrm{~Hz}, 1 \mathrm{H}) \cdot{ }^{13} \mathrm{C}$ NMR $\left(101 \mathrm{MHz}, \mathrm{CDCl}_{3}\right): \delta=25.3\left(\mathrm{CH}_{2}\right), 29.8$ $\left(\mathrm{CH}_{3}\right), 39.6\left(\mathrm{CH}_{2}\right), 43.2\left(\mathrm{CH}_{3}\right), 110.1,114.0,120.4,120.6,124.0,124.4,125.4,127.0$, 128.4, 131.3, 134.3, 136.3, $167.7(\mathrm{C}=\mathrm{O}) . \mathrm{IR} v\left(\mathrm{~cm}^{-1}\right): 3308(\mathrm{~N}-\mathrm{H}), 2932(\mathrm{Ar}-\mathrm{H}), 1630$ $(\mathrm{C}=\mathrm{O}), 1543,1472,1355,1311,1164,962,874,748$. HRMS (ESI-TOF): calcd. for $\mathrm{C}_{20} \mathrm{H}_{23} \mathrm{~N}_{3} \mathrm{NaO}_{5} \mathrm{~S}_{2}$ : 472.0971; found: 472.0979 .

\section{$N$-(2-(5-(Benzyloxy)-1-methyl-2-( $N$-(methylsulfonyl)methylsulfonamido)-1 $H$-indol-3-}

yl)ethyl)benzamide 8i. $89 \mathrm{mg}, 80 \%$ yield, brown solid. m.p. $=157-159{ }^{\circ} \mathrm{C} .{ }^{1} \mathrm{H}$ NMR $(400$ $\left.\mathrm{MHz}, \mathrm{CDCl}_{3}\right): \delta=3.12(\mathrm{t}, J=6.9 \mathrm{~Hz}, 2 \mathrm{H}), 3.50(\mathrm{~s}, 6 \mathrm{H}), 3.70(\mathrm{~s}, 3 \mathrm{H}), 3.89-3.94(\mathrm{~m}, 2 \mathrm{H})$, $5.03(\mathrm{~s}, 2 \mathrm{H}), 6.55$ (t, $J=5.5 \mathrm{~Hz}, 1 \mathrm{H}), 7.08(\mathrm{dd}, J=9.0,2.4 \mathrm{~Hz}, 1 \mathrm{H}), 7.23-7.27$ (m, 2H), 7.31-7.45 (m, 8H), 7.70-7.72 (m, 2H). ${ }^{13} \mathrm{C}$ NMR (101 MHz, $\left.\mathrm{CDCl}_{3}\right): \delta=25.1\left(\mathrm{CH}_{2}\right), 30.0$ $\left(\mathrm{CH}_{3}\right), 39.5\left(\mathrm{CH}_{2}\right), 43.2\left(\mathrm{CH}_{3}\right), 70.8\left(\mathrm{CH}_{2}\right), 103.2,111.2,113.5,115.9,124.1,125.6,127.1$, 
$127.7,127.9,128.4,128.5,131.4,131.7,134.3,137.2,153.6,167.7(\mathrm{C}=\mathrm{O}) . \quad \mathrm{IR} v\left(\mathrm{~cm}^{-1}\right)$ :

3341 (N-H), 3062 (Ar-H), 3033 (Ar-H), 2930 (Ar-H), 1711 (C=O), 1651, 1532, 1488, 1451, 1366, 1279, 1162, 1128, 1022, 875, 727, 694. HRMS (ESI-TOF): calcd. for $\mathrm{C}_{27} \mathrm{H}_{29} \mathrm{~N}_{3} \mathrm{NaO}_{6} \mathrm{~S}_{2}$ : 578.1390; found: 578.1403 .

\section{$\mathrm{N}$-(2-(5-Iodo-2-(4-methyl- $\mathrm{N}$-tosylphenylsulfonamido)-1 $\mathrm{H}$-indol-3-yl)ethyl)acetamide 9.}

To a stirred solution of acetamide $7 \mathbf{a}(47.8 \mathrm{mg}, 0.091 \mathrm{mmol}$, 1equiv) and TFA (2 mL) in $\mathrm{CH}_{2} \mathrm{Cl}_{2}(20 \mathrm{~mL})$ at $-78{ }^{\circ} \mathrm{C}, \mathrm{HBF}_{4} \cdot \mathrm{Et}_{2} \mathrm{O}(0.038 \mathrm{~mL}, 0.3 \mathrm{mmol}, 3$ equiv $)$ and bis(pyridine)iodonium tetrafluoroborate $(0.034 \mathrm{~g}, 0.091 \mathrm{mmol}, 1$ equiv) were added. The brown solution was stirred for $2 \mathrm{~h}$. The reaction was quenched with cold water. The two phases were separated and the organic layer was washed with water and an aqueous saturated solution of sodium thiosulfate. The organic phase was dried over $\mathrm{Na}_{2} \mathrm{SO}_{4}$ and the solvent was removed under reduced pressure to obtain a crude product, which was purified by column chromatography on silica gel ( $n$-hexane/EtOAc, $1 / 1, v / v)$ to afford the pure product (46 mg, 78\% yield, white solid). m.p. $=187-191{ }^{\circ} \mathrm{C} .{ }^{1} \mathrm{H}$ NMR $\left(400 \mathrm{MHz}, \mathrm{CD}_{2} \mathrm{Cl}_{2}\right)$ : $\delta=1.79(\mathrm{~s}, 3 \mathrm{H}), 2.15(\mathrm{t}, J=6.7 \mathrm{~Hz}, 2 \mathrm{H}), 2.48(\mathrm{~s}, 6 \mathrm{H}), 3.35(\mathrm{td}, J=6.2,5.7 \mathrm{~Hz}, 2 \mathrm{H}), 7.17$ $(\mathrm{d}, J=8.7 \mathrm{~Hz}, 1 \mathrm{H}), 7.39(\mathrm{~d}, J=7.8 \mathrm{~Hz}, 4 \mathrm{H}), 7.52(\mathrm{dd}, J=8.6,1.6 \mathrm{~Hz}, 1 \mathrm{H}), 7.80(\mathrm{~d}, J=7.8$ $\mathrm{Hz}, 4 \mathrm{H}), 7.96(\mathrm{~s}, 1 \mathrm{H}) .{ }^{13} \mathrm{C}$ NMR $\left(101 \mathrm{MHz}, \mathrm{CD}_{2} \mathrm{Cl}_{2}\right): \delta=21.9\left(\mathrm{CH}_{3}\right), 22.9\left(\mathrm{CH}_{3}\right), 23.4$ $\left(\mathrm{CH}_{2}\right), 39.1\left(\mathrm{CH}_{2}\right), 83.4$ (C-I), 114.3, 115.0, 124.2, 129.0, 129.1, 129.4, 130.3, 132.8, 134.6, 136.1, 146.7, $171.6(\mathrm{C}=\mathrm{O})$. IR $v\left(\mathrm{~cm}^{-1}\right): 3379(\mathrm{~N}-\mathrm{H}), 3170(\mathrm{Ar}-\mathrm{H}), 2921(\mathrm{Ar}-\mathrm{H}), 2582($ ArH), $2366(\mathrm{Ar}-\mathrm{H}), 1643(\mathrm{C}=\mathrm{O}), 1596,1376,1353,1165,879,660,542$. HRMS (ESI-TOF): calcd. for $\mathrm{C}_{26} \mathrm{H}_{26} \mathrm{IN}_{3} \mathrm{O}_{5} \mathrm{NaS}_{2}$ : 674.0256, found: 674.0279. 


\section{$\mathrm{N}$-(2-(5-Iodo-1-methyl-2-(4-methyl- $\mathrm{N}$-tosylphenylsulfonamido)-1H-indol-3-}

yl)ethyl)acetamide 10. Synthesized from 4a through an identical procedure as described for compound 9. $59 \mathrm{mg}$, 98\% yield, white solid. m.p. $=196-197{ }^{\circ} \mathrm{C} .{ }^{1} \mathrm{H} \mathrm{NMR}(500 \mathrm{MHz}$, $\left.\mathrm{CDCl}_{3}\right): \delta=1.82(\mathrm{~s}, 3 \mathrm{H}), 2.15(\mathrm{t}, J=6.4 \mathrm{~Hz}, 2 \mathrm{H}), 2.50(\mathrm{~s}, 6 \mathrm{H}), 3.31(\mathrm{~s}, 3 \mathrm{H}), 3.48-3.52(\mathrm{~m}$, 2H), $6.11(\mathrm{t}, J=6.1 \mathrm{~Hz}, 1 \mathrm{H}), 7.07(\mathrm{~d}, J=8.7 \mathrm{~Hz}, 1 \mathrm{H}), 7.38(\mathrm{~d}, J=8.1 \mathrm{~Hz}, 4 \mathrm{H}), 7.58(\mathrm{dd}, J$ $=8.8,1.6 \mathrm{~Hz}, 1 \mathrm{H}), 7.85(\mathrm{~d}, J=8.4 \mathrm{~Hz}, 4 \mathrm{H}), 7.98(\mathrm{~s}, 1 \mathrm{H}) .{ }^{13} \mathrm{C} \mathrm{NMR}\left(125 \mathrm{MHz}, \mathrm{CDCl}_{3}\right): \delta=$ $21.8\left(\mathrm{CH}_{3}\right), 23.0\left(\mathrm{CH}_{3}\right), 23.5\left(\mathrm{CH}_{2}\right), 30.0\left(\mathrm{CH}_{3}\right), 38.9\left(\mathrm{CH}_{2}\right), 83.4(\mathrm{C}-\mathrm{I}), 112.2,114.1$, $125.7,127.8,129.1,129.3,129.9,132.4,135.2,135.5,146.13,170.3(\mathrm{C}=\mathrm{O}) . \mathrm{IR} \vee\left(\mathrm{cm}^{-1}\right)$ : 3290 (N-H), 2923 (Ar-H), 2853 (Ar-H), 1651 (C=O), 1372, 1166, 873, 659, 542, 478. HRMS (ESI-TOF): calcd. for $\mathrm{C}_{27} \mathrm{H}_{28} \mathrm{IN}_{3} \mathrm{O}_{5} \mathrm{NaS}_{2}$ : 688.0413, found: 688.0437.

\section{$N$-(2-(5,6-Diiodo-2-(4-methyl- $N$-tosylphenylsulfonamido)-1 $H$-indol-3-}

yl)ethyl)acetamide 11 and $N$-(2-(5,7-Diiodo-2-(4-methyl-N-tosylphenylsulfonamido)-

1H-indol-3-yl)ethyl)acetamide 12. To a stirred solution of the acetamide $7 \mathbf{a}(0.052 \mathrm{~g}$, 0.1mmol, 1 equiv) and TFA $(2 \mathrm{~mL})$ in $\mathrm{CH}_{2} \mathrm{Cl}_{2}(20 \mathrm{~mL})$ at $-78{ }^{\circ} \mathrm{C}, \mathrm{HBF}_{4} \cdot \mathrm{Et}_{2} \mathrm{O}(0.086 \mathrm{~mL}$, $0.6 \mathrm{mmol}, 6$ equiv) and bis(pyridine)iodonium tetrafluoroborate $(0.082 \mathrm{~g}, 0.22 \mathrm{mmol}, 2.2$ equiv) were added. The brown solution was allowed to stir for $2 \mathrm{~h}$. The reaction was quenched with cold water, after extraction the organic layer was washed with an aqueous saturated solution of $\mathrm{Na}_{2} \mathrm{~S}_{2} \mathrm{O}_{4}$. The combined organic phases were dried over $\mathrm{Na}_{2} \mathrm{SO}_{4}$ and the solvent was removed under reduced pressure. The residue was purified by column chromatography (silica gel, $\mathrm{CH}_{2} \mathrm{Cl}_{2} /$ EtOAc, 1/1, v/v) to afford a mixture of the two regioisomers in 1:3-ratio. (53 mg, 68\% yield, white solids. ${ }^{1} \mathrm{H}$ NMR (400 $\mathrm{MHz}, \mathrm{CDCl}_{3}$ ): $\delta$ $=1.86(\mathrm{~s}, 6 \mathrm{H}), 2.12-2.15(\mathrm{~m}, 2 \mathrm{H}), 2.19-2.22(\mathrm{~m}, 2 \mathrm{H}), 2.52(\mathrm{~s}, 6 \mathrm{H}), 2.53(\mathrm{~s}, 6 \mathrm{H}), 3.43-3.48$ 
$(\mathrm{m}, 2 \mathrm{H}), 3.64-3.68(\mathrm{~m}, 2 \mathrm{H}), 6.12(\mathrm{~s}, 2 \mathrm{H}), 7.39-7.44(\mathrm{~m}, 8 \mathrm{H}), 7.84-7.89(\mathrm{~m}, 8 \mathrm{H}), 7.93(\mathrm{~s}$, 1H), $7.95(\mathrm{~s}, 1 \mathrm{H}), 8.17(\mathrm{~s}, 1 \mathrm{H}), 8.18(\mathrm{~s}, 1 \mathrm{H}) .{ }^{13} \mathrm{C} \mathrm{NMR}\left(101 \mathrm{MHz}, \mathrm{CDCl}_{3}\right): \delta=21.8\left(\mathrm{CH}_{3}\right)$, $22.7\left(\mathrm{CH}_{2}\right), 22.8\left(\mathrm{CH}_{2}\right), 23.0\left(\mathrm{CH}_{3}\right), 31.9\left(\mathrm{CH}_{2}\right), 38.7\left(\mathrm{CH}_{2}\right), 96.9(\mathrm{C}-\mathrm{I}), 101.7(\mathrm{C}-\mathrm{I}), 115.4$, $122.4,124.5,128.5,128.7,128.8,130.0,130.1,130.5,135.4,135.5,146.1,146.3,170.5$ $(\mathrm{C}=\mathrm{O}) . \mathrm{IR} \vee\left(\mathrm{cm}^{-1}\right): 3387(\mathrm{~N}-\mathrm{H}), 2919(\mathrm{Ar}-\mathrm{H}), 2850(\mathrm{Ar}-\mathrm{H}), 1657(\mathrm{C}=\mathrm{O}), 1597,1540$ 1375, 1351, 1164, 1083, 876, 754, 659, 541. HRMS (ESI-TOF): calcd. for $\mathrm{C}_{26} \mathrm{H}_{26} \mathrm{I}_{2} \mathrm{~N}_{3} \mathrm{O}_{5} \mathrm{~S}_{2}$, 777.9398, found: 777.9418 .

General procedure for fluorination. Selectfluor ${ }^{\circledR}(0.019 \mathrm{~g}, 0.06 \mathrm{mmol}, 1.2$ equiv) was added to a stirred solution of the corresponding starting material $(0.027 \mathrm{~g}, 0.05 \mathrm{mmol}, 1.0$ equiv) and $\mathrm{NaHCO}_{3}(0.005 \mathrm{~g}, 0.06 \mathrm{mmol}, 1.2$ equiv $)$ in acetonitrile at $70{ }^{\circ} \mathrm{C}$. The reaction was allowed to stir at $70{ }^{\circ} \mathrm{C}$ for $16 \mathrm{~h}$. The solvent was removed under reduced pressure and an aqueous saturated solution of $\mathrm{NaHCO}_{3}$ was added. The residue was extracted with ethyl acetate and the combined organic phases were dried over $\mathrm{Na}_{2} \mathrm{SO}_{4}$, filtered and the solvent was removed under reduced pressure. The residue was purified by column chromatography ( silica gel, $n$-hexane/EtOAc, 2/3, v/v). The products were obtained as solids in 95-99\% isolated yield.

N-(2-(3-Fluoro-2-(tosylimino)indolin-3-yl)ethyl)acetamide 13a. $19 \mathrm{mg}, 98 \%$ yield, yellow solid. m.p. $=230-232{ }^{\circ} \mathrm{C} .{ }^{1} \mathrm{H}$ NMR $\left(500 \mathrm{MHz}, \mathrm{CDCl}_{3}\right): \delta=1.87(\mathrm{~s}, 3 \mathrm{H}), 2.27-2.50$ (m, 5H), $3.33(\mathrm{~m}, 2 \mathrm{H}), 5.93(\mathrm{brs}, 1 \mathrm{H}), 7.02(\mathrm{~d}, J=7.9 \mathrm{~Hz}, 1 \mathrm{H}), 7.15(\mathrm{t}, J=7.6 \mathrm{~Hz}, 1 \mathrm{H})$, 7.31-7.37 (m, 3H), $7.42(\mathrm{~d}, J=7.5 \mathrm{~Hz}, 1 \mathrm{H}) 7.88(\mathrm{~d}, J=8.3 \mathrm{~Hz}, 2 \mathrm{H}), 9.82(\mathrm{~s}, 1 \mathrm{H}) .{ }^{13} \mathrm{C} \mathrm{NMR}$ $\left(125 \mathrm{MHz}, \mathrm{CDCl}_{3}\right): \delta=21.6\left(\mathrm{CH}_{3}\right), 23.1\left(\mathrm{CH}_{3}\right), 33.7\left(\mathrm{~d}, J_{C-F}=9.8 \mathrm{~Hz}, \mathrm{CH}_{2}\right), 35.8\left(\mathrm{~d}, J_{C-F}=\right.$ $\left.27.9 \mathrm{~Hz}, \mathrm{CH}_{2}\right), 97.3\left(\mathrm{~d}, J_{C-F}=192.2 \mathrm{~Hz}, \mathrm{C}-\mathrm{F}\right), 111.8,124.6\left(\mathrm{~d}, J_{C-F}=2.7 \mathrm{~Hz}\right), 124.8,125.6$ 
$\left(\mathrm{d}, J_{C-F}=18.7 \mathrm{~Hz}\right), 126.8,129.7,131.9(\mathrm{~d}, J=3.1 \mathrm{~Hz}), 137.5,141.0,144.2,165.1\left(\mathrm{~d}, J_{C-F}=\right.$ $19.7 \mathrm{~Hz}), 170.3(\mathrm{C}=\mathrm{O}) .{ }^{19} \mathrm{~F}$ NMR $\left(376 \mathrm{MHz}, \mathrm{CDCl}_{3}\right): \delta=-150.3 . \operatorname{IR} v\left(\mathrm{~cm}^{-1}\right): 3303(\mathrm{~N}-\mathrm{H})$, $2921($ Ar-H), 2851 (Ar-H), $1615(\mathrm{C}=\mathrm{O})$, 1469, 1284, 1143, 1082, 752, 665, 546. HRMS (ESI-TOF): calcd. for $\mathrm{C}_{19} \mathrm{H}_{19} \mathrm{FN}_{3} \mathrm{O}_{3} \mathrm{~S}$ : 388.1137, found: 388.1137 .

$N$-(2-(3-Fluoro-2-(tosylimino)indolin-3-yl)ethyl)benzamide 13b. $21 \mathrm{mg}, 95 \%$ yield, yellow solid. m.p. $=202-204{ }^{\circ} \mathrm{C} .{ }^{1} \mathrm{H}$ NMR $\left(400 \mathrm{MHz}, \mathrm{CDCl}_{3}\right): \delta=2.33-2.64(\mathrm{~m}, 5 \mathrm{H}), 3.62$ (td, $J=6.4,6.2 \mathrm{~Hz}, 2 \mathrm{H}), 6.52(\mathrm{t}, J=5.8 \mathrm{~Hz}, 1 \mathrm{H}), 7.00(\mathrm{~d}, J=7.8 \mathrm{~Hz}, 1 \mathrm{H}), 7.16-7.19(\mathrm{~m}$, 1H), $7.30(\mathrm{~d}, J=8.2 \mathrm{~Hz}, 2 \mathrm{H}), 7.36-7.49(\mathrm{~m}, 5 \mathrm{H}), 7.69-7.72(\mathrm{~m}, 2 \mathrm{H}), 7.86(\mathrm{~d}, J=8.3 \mathrm{~Hz}$, 2H), $9.82(\mathrm{~s}, 1 \mathrm{H}) .{ }^{13} \mathrm{C} \mathrm{NMR}\left(101 \mathrm{MHz}, \mathrm{CDCl}_{3}\right): \delta=21.6\left(\mathrm{CH}_{3}\right), 34.2\left(\mathrm{~d}, J_{C-F}=10.1 \mathrm{~Hz}\right.$, $\left.\mathrm{CH}_{2}\right), 35.6\left(\mathrm{~d}, J_{C-F}=27.2 \mathrm{~Hz}, \mathrm{CH}_{2}\right), 99.2\left(\mathrm{~d}, J_{C-F}=194.5 \mathrm{~Hz}, \mathrm{C}-\mathrm{F}\right) 111.8,124.7\left(\mathrm{~d}, J_{C-F}=\right.$ $2.7 \mathrm{~Hz}), 124.9,126.8\left(\mathrm{~d}, J_{C-F}=16.7 \mathrm{~Hz}\right), 129.7,131.5,131.9,132.0,134.0,137.6,141.0$, 144.1, $164.9\left(\mathrm{~d}, J_{C-F}=24.3 \mathrm{~Hz}\right), 167.3(\mathrm{C}=\mathrm{O}) .{ }^{19} \mathrm{~F} \mathrm{NMR}\left(376 \mathrm{MHz}, \mathrm{CDCl}_{3}\right): \delta=-149.8 . \mathrm{IR}$ $v\left(\mathrm{~cm}^{-1}\right): 3295(\mathrm{~N}-\mathrm{H}), 2851(\mathrm{Ar}-\mathrm{H}), 1616(\mathrm{C}=\mathrm{O}), 1532,1470,1284,1142,1082,669,547$. HRMS (ESI-TOF): calcd. for $\mathrm{C}_{24} \mathrm{H}_{22} \mathrm{FN}_{3} \mathrm{NaO}_{3} \mathrm{~S}: 474.1264$, found: 474.1260 .

$N$-(2-(3-Fluoro-1-methyl-2-(tosylimino)indolin-3-yl)ethyl)acetamide 14a. 20 mg, 99\% yield, yellow solid. m.p. $=47-53{ }^{\circ} \mathrm{C} .{ }^{1} \mathrm{H}$ NMR $\left(500 \mathrm{MHz}, \mathrm{CDCl}_{3}\right): \delta=1.87(\mathrm{~s}, 3 \mathrm{H}), 2.44(\mathrm{~s}$, $3 \mathrm{H}), 2.64-2.70(\mathrm{~m}, 1 \mathrm{H}), 3.08-3.13(\mathrm{~m}, 1 \mathrm{H}), 3.20-3.38(\mathrm{~m}, 5 \mathrm{H}), 6.32(\mathrm{~s}, 1 \mathrm{H}), 6.90-6.92(\mathrm{~m}$, 1H), $7.19(\mathrm{t}, J=7.6 \mathrm{~Hz}, 1 \mathrm{H}), 7.31(\mathrm{~d}, J=8.0 \mathrm{~Hz}, 2 \mathrm{H}), 7.41-7.44(\mathrm{~m}, 2 \mathrm{H}), 7.92(\mathrm{~d}, J=8.3$ $\mathrm{Hz}, 2 \mathrm{H}) .{ }^{13} \mathrm{C}$ NMR $\left(125 \mathrm{MHz}, \mathrm{CDCl}_{3}\right): \delta=21.6\left(\mathrm{CH}_{3}\right), 23.1\left(\mathrm{CH}_{3}\right), 29.0\left(\mathrm{CH}_{3}\right), 33.9(\mathrm{~d}$, $\left.J_{C-F}=9.8 \mathrm{~Hz}, \mathrm{CH}_{2}\right), 35.6\left(\mathrm{~d}, J_{C-F}=27.1 \mathrm{~Hz}, \mathrm{CH}_{2}\right), 96.9\left(\mathrm{~d}, J_{C-F}=194.3 \mathrm{~Hz}, \mathrm{C}-\mathrm{I}\right), 110.0$, $124.1,124.7\left(\mathrm{~d}, J_{C-F}=2.7 \mathrm{~Hz}\right), 126.7,127.1\left(\mathrm{~d}, J_{C-F}=19.0 \mathrm{~Hz}\right), 129.4,131.8\left(\mathrm{~d}, J_{C-F}=2.7\right.$ 
$\mathrm{Hz}), 140.0,143.0,143.3\left(\mathrm{~d}, J_{C-F}=5.1 \mathrm{~Hz}\right), 164.8\left(\mathrm{~d}, J_{C-F}=23.5 \mathrm{~Hz}\right), 170.1(\mathrm{C}=\mathrm{O}) .{ }^{19} \mathrm{~F}$ NMR (376 MHz, $\left.\mathrm{CDCl}_{3}\right): \delta=-153.0$. IR $v\left(\mathrm{~cm}^{-1}\right): 3294(\mathrm{~N}-\mathrm{H}), 2927(\mathrm{Ar}-\mathrm{H}), 1632(\mathrm{C}=\mathrm{O})$, 1580, 1470, 1282, 1145, 1082, 770, 751, 671, 545, 461. HRMS (ESI-TOF): calcd. for $\mathrm{C}_{20} \mathrm{H}_{22} \mathrm{FN}_{3} \mathrm{O}_{3} \mathrm{NaS}$ : 426.1258; found: 426.1261 .

$N$-(2-(3-Fluoro-1-methyl-2-(tosylimino)indolin-3-yl)ethyl)benzamide 14b. $22 \mathrm{mg}, 98 \%$ yield, orange solid. m.p. $=128-129^{\circ} \mathrm{C} .{ }^{1} \mathrm{H}$ NMR $\left(400 \mathrm{MHz}, \mathrm{CDCl}_{3}\right) \delta=2.46(\mathrm{~s}, 3 \mathrm{H}), 2.73-$ $2.80(\mathrm{~m}, 1 \mathrm{H}), 3.02(\mathrm{~s}, 3 \mathrm{H}), 3.30-3.36(\mathrm{~m}, 1 \mathrm{H}), 3.50-3.71(\mathrm{~m}, 2 \mathrm{H}), 6.90(\mathrm{~d}, J=7.9 \mathrm{~Hz}, 1 \mathrm{H})$, 7.12-7.14 (m, 1H), $7.20(\mathrm{t}, J=7.6 \mathrm{~Hz}, 1 \mathrm{H}), 7.29-7.32(\mathrm{~m}, 2 \mathrm{H}), 7.38-7.52(\mathrm{~m}, 5 \mathrm{H}), 7.84-$

$7.88(\mathrm{~m}, 4 \mathrm{H}) .{ }^{13} \mathrm{C} \mathrm{NMR}\left(101 \mathrm{MHz}, \mathrm{CDCl}_{3}\right) \delta=21.5\left(\mathrm{CH}_{3}\right), 28.7\left(\mathrm{CH}_{3}\right), 34.5\left(\mathrm{~d}, J_{C-F}=\right.$ $10.1 \mathrm{~Hz}, \mathrm{CH}_{2}$ ), 35.3 (d, $\left.J_{C-F}=27.4 \mathrm{~Hz}, \mathrm{CH}_{2}\right), 96.9$ (d, $\left.J_{C-F}=193.6 \mathrm{~Hz}, \mathrm{C}-\mathrm{I}\right), 110.0,124.0$, $124.7(\mathrm{~d}, J=2.7 \mathrm{~Hz}), 126.6,126.8,127.0,127.2,128.4,129.3,131.4,131.8\left(\mathrm{~d}, J_{C-F}=2.8\right.$ $\mathrm{Hz}), 133.8,140.0,142.9,143.3\left(\mathrm{~d}, J_{C-F}=5.2 \mathrm{~Hz}\right), 165.3\left(\mathrm{~d}, J_{C-F}=23.5 \mathrm{~Hz}\right), 166.8(\mathrm{C}=\mathrm{O})$.

${ }^{19} \mathrm{~F}$ NMR $\left(376 \mathrm{MHz}, \mathrm{CDCl}_{3}\right) \delta=-152.8$. IR $v\left(\mathrm{~cm}^{-1}\right): 3253(\mathrm{~N}-\mathrm{H}), 3068(\mathrm{Ar}-\mathrm{H}), 2920(\mathrm{Ar}-$ H), $1627(\mathrm{C}=\mathrm{O})$, 1550, 1381, 1167, 876, 657, 540. HRMS (ESI-TOF): calcd for $\mathrm{C}_{25} \mathrm{H}_{24} \mathrm{FN}_{3} \mathrm{NaO}_{3} \mathrm{~S}$ : 488.1415; found: 488.1422 .

\section{$N$-(2-(3-Fluoro-2-(4-methyl- $N$-tosylphenylsulfonamido)-3H-indol-3-yl)ethyl)acetamide}

15. $23 \mathrm{mg}, 86 \%$ yield, yellow solid. m.p. $=132-134{ }^{\circ} \mathrm{C} .{ }^{1} \mathrm{H}$ NMR $\left(400 \mathrm{MHz}, \mathrm{CDCl}_{3}\right): \delta=$ $1.90(\mathrm{~s}, 3 \mathrm{H}), 2.13-2.25(\mathrm{~m}, 1 \mathrm{H}), 2.26-2.40(\mathrm{~m}, 1 \mathrm{H}), 2.51(\mathrm{~s}, 6 \mathrm{H}), 3.15-3.23(\mathrm{~m}, 1 \mathrm{H}), 3.34-$ $3.43(\mathrm{~m}, 1 \mathrm{H}), 5.51(\mathrm{t}, J=6.0,1 \mathrm{H}), 7.36-7.41(\mathrm{~m}, 5 \mathrm{H}), 7.44-7.52(\mathrm{~m}, 2 \mathrm{H}), 7.57(\mathrm{~d}, J=7.6$ $\mathrm{Hz}, 1 \mathrm{H}), 8.11(\mathrm{~d}, J=8.4,4 \mathrm{H}) .{ }^{13} \mathrm{C}$ NMR $\left(101 \mathrm{MHz}, \mathrm{CDCl}_{3}\right): \delta=21.8\left(\mathrm{CH}_{3}\right), 23.2\left(\mathrm{CH}_{3}\right)$, $33.5\left(\mathrm{~d}, J_{C-F}=26.0 \mathrm{~Hz}, \mathrm{CH}_{2}\right), 34.0\left(\mathrm{~d}, J_{C-F}=6.9 \mathrm{~Hz}, \mathrm{CH}_{2}\right), 100.4\left(\mathrm{~d}, J_{C-F}=197.0 \mathrm{~Hz}, \mathrm{C}-\mathrm{F}\right)$, 
101.4, 122.9, 123.9, $128.8\left(\mathrm{~d}, J_{C-F}=1.6 \mathrm{~Hz}\right), 129.5,129.7\left(\mathrm{~d}, J_{C-F}=1.9 \mathrm{~Hz}\right), 131.2,134.4$ $\left(\mathrm{d}, J_{C-F}=18.5 \mathrm{~Hz}\right), 136.2,145.8,149.7\left(\mathrm{~d}, J_{C-F}=6.3 \mathrm{~Hz}\right), 165.4\left(\mathrm{~d}, J_{C-F}=18.8 \mathrm{~Hz}\right), 169.8$ $(\mathrm{C}=\mathrm{O}) .{ }^{19} \mathrm{~F}$ NMR $\left(376 \mathrm{MHz}, \mathrm{CDCl}_{3}\right): \delta=-166.6 . \mathrm{IR} v\left(\mathrm{~cm}^{-1}\right): 3282(\mathrm{~N}-\mathrm{H}), 2919(\mathrm{Ar}-\mathrm{H})$, $2850($ Ar-H), $1650(\mathrm{C}=\mathrm{O}), 1563,1381,1167,1082,886,659,538$. HRMS (ESI-TOF): calcd. for $\mathrm{C}_{26} \mathrm{H}_{25} \mathrm{FN}_{3} \mathrm{O}_{5} \mathrm{~S}_{2}:$ 542.1225, found: 542.1221.

$N$-(2-(3-Fluoro-1-methyl-2-oxoindolin-3-yl)ethyl)acetamide 16a. Compound 14a (0.045 $\mathrm{g}, 0.11 \mathrm{mmol}, 1$ equiv) was dissolved in $\mathrm{MeOH}(2 \mathrm{~mL})$ and $\mathrm{LiOH}(0.027,1.1 \mathrm{mmol}, 10$ equiv) was added. The reaction mixture was stirred at $25{ }^{\circ} \mathrm{C}$ for $16 \mathrm{~h}$. The solvent was removed under reduced pressure. The residue was quenched with water and extracted with $\mathrm{CH}_{2} \mathrm{Cl}_{2}$. The organic layer was dried over $\mathrm{Na}_{2} \mathrm{SO}_{4}$, filtered and the solvent was removed under reduced pressure. The residue was purified by column chromatography (silica gel, $\left.\mathrm{MeOH} / \mathrm{CH}_{2} \mathrm{Cl}_{2}, 0.2: 10, \mathrm{v} / \mathrm{v}\right)$ to afford the pure product in $85 \%$ yield $(0.023 \mathrm{~g})$ as colorless oil. ${ }^{1} \mathrm{H}$ NMR (400 MHz, $\left.\mathrm{CDCl}_{3}\right): \delta=1.94(\mathrm{~s}, 3 \mathrm{H}), 2.14-2.27(\mathrm{~m}, 1 \mathrm{H}), 2.41-2.52(\mathrm{~m}, 1 \mathrm{H})$, $3.22(\mathrm{~s}, 3 \mathrm{H}), 3.43-3.60(\mathrm{~m}, 2 \mathrm{H}), 6.15$ (brs, $1 \mathrm{H}), 6.87$ (d, $J=8.3 \mathrm{~Hz}, 1 \mathrm{H}), 7.15(\mathrm{t}, J=7.9 \mathrm{~Hz}$, 1H), 7.40-7.44 (m, 2H). ${ }^{13} \mathrm{C}$ NMR $\left(101 \mathrm{MHz}, \mathrm{CDCl}_{3}\right): \delta=23.2\left(\mathrm{CH}_{3}\right), 26.3\left(\mathrm{CH}_{3}\right), 33.9(\mathrm{~d}$, $\left.J_{C-F}=5.9 \mathrm{~Hz}, \mathrm{CH}_{2}\right), 34.8\left(\mathrm{~d}, J_{C-F}=26.8 \mathrm{~Hz}\right), 92.4\left(\mathrm{~d}, J_{C-F}=186.9 \mathrm{~Hz}, \mathrm{CH}_{2}-\mathrm{F}\right), 108.9,123.6$ $\left(\mathrm{d}, J_{C-F}=2.8 \mathrm{~Hz}\right), 124.5,125.9\left(\mathrm{~d}, J_{C-F}=18.2 \mathrm{~Hz}\right), 126.4,129.7,131.5\left(\mathrm{~d}, J_{C-F}=3.0 \mathrm{~Hz}\right)$, $143.7\left(\mathrm{~d}, J_{C-F}=5.4 \mathrm{~Hz}\right), 170.0(\mathrm{C}=\mathrm{O}), 172.8\left(\mathrm{~d}, J_{C-F}=22.0 \mathrm{~Hz}, \mathrm{C}=\mathrm{O}\right) .{ }^{19} \mathrm{~F}$ NMR $(101 \mathrm{MHz}$, $\left.\mathrm{CDCl}_{3}\right): \delta=-158.5 . \mathrm{IR} v\left(\mathrm{~cm}^{-1}\right): 3294(\mathrm{~N}-\mathrm{H}), 3065(\mathrm{Ar}-\mathrm{H}), 2927(\mathrm{Ar}-\mathrm{H}), 1724(\mathrm{C}=\mathrm{O}), 1615$ $(\mathrm{C}=\mathrm{O}), 1470,1372,1246,1113,750$. HRMS (ESI-TOF): calcd. for $\mathrm{C}_{13} \mathrm{H}_{15} \mathrm{FN}_{2} \mathrm{NaO}_{2}$ : 273.1010, found: 273.1013 . 
$N$-(2-(3-Fluoro-1-methyl-2-oxoindolin-3-yl)ethyl)benzamide 16b. Synthesized in an analogous manner to compound 16a as described above. $29 \mathrm{mg}$, 85\% yield, colorless oil. ${ }^{1} \mathrm{H}$ NMR (400 MHz, $\left.\mathrm{CDCl}_{3}\right): \delta=2.23-2.36(\mathrm{~m}, 1 \mathrm{H}), 2.57-2.67(\mathrm{~m}, 1 \mathrm{H}), 3.18(\mathrm{~s}, 3 \mathrm{H}), 3.63-$ $3.71(\mathrm{~m}, 1 \mathrm{H}), 3.78-3.87(\mathrm{~m}, 1 \mathrm{H}), 6.86(\mathrm{~d}, J=7.8 \mathrm{~Hz}, 1 \mathrm{H}), 7.02(\mathrm{brs}, 1 \mathrm{H}), 7.16(\mathrm{t}, J=7.6$ $\mathrm{Hz}, 1 \mathrm{H}), 7.39-7.52(\mathrm{~m}, 5 \mathrm{H}), 7.76-7.82(\mathrm{~m}, 2 \mathrm{H}) .{ }^{13} \mathrm{C} \mathrm{NMR}\left(101 \mathrm{MHz}, \mathrm{CDCl}_{3}\right): \delta=26.3$ $\left(\mathrm{CH}_{3}\right), 34.3\left(\mathrm{~d}, J_{C-F}=5.8 \mathrm{~Hz}, \mathrm{CH}_{2}\right), 34.8\left(\mathrm{~d}, J_{C-F}=27.0 \mathrm{~Hz}, \mathrm{CH}_{2}\right), 92.5\left(\mathrm{~d}, J_{C-F}=186.8 \mathrm{~Hz}\right.$ C-F), 109.0, $123.7\left(\mathrm{~d}, J_{C-F}=2.7 \mathrm{~Hz}\right), 124.5,125.9\left(\mathrm{~d}, J_{C-F}=18.3 \mathrm{~Hz}\right), 126.9,128.5,131.4$, $131.6\left(\mathrm{~d}, J_{C-F}=3.2 \mathrm{~Hz}\right), 134.3,143.6\left(\mathrm{~d}, J_{C-F}=5.4 \mathrm{~Hz}\right), 167.0(\mathrm{C}=\mathrm{O}), 173.0\left(\mathrm{~d}, J_{C-F}=22.0\right.$ $\mathrm{Hz}, \mathrm{C}=\mathrm{O}) .{ }^{19} \mathrm{~F}$ NMR $\left(101 \mathrm{MHz}, \mathrm{CDCl}_{3}\right): \delta=-158.3 . \mathrm{IR} v\left(\mathrm{~cm}^{-1}\right): 3327(\mathrm{~N}-\mathrm{H}), 2923(\mathrm{Ar}-\mathrm{H})$, $2852(\mathrm{Ar}-\mathrm{H}), 1725(\mathrm{C}=\mathrm{O}), 1639(\mathrm{C}=\mathrm{O}), 1616,1537,1491,1470,1376,1306,1092,751$, 694. HRMS (ESI-TOF): calcd. for $\mathrm{C}_{18} \mathrm{H}_{17} \mathrm{FN}_{2} \mathrm{NaO}_{2}$ : 335.1166 , found: 335.1169 .

General procedure for treatment of $13 \mathbf{a}, \mathrm{b}$ and $14 \mathrm{a}, \mathrm{b}$ with TFA. The corresponding starting material (0.12 mmol, 1 equiv) was dissolved in TFA (4 mL) and the reaction mixture was allowed to stir for $12 \mathrm{~h}$ under an atmosphere of argon. The solvent was removed under reduced pressure and the pure product was isolated in quantitative yield.

2-(3-Acetoxy-2-(tosylimino)indolin-3-yl)ethanaminium trifluoroacetate 17a. 59 mg, 98\% yield, brown solid. m.p. $=112-115{ }^{\circ} \mathrm{C} .{ }^{1} \mathrm{H}$ NMR (400 MHz, MeOD): $\delta=2.43$ (s, 3H), $2.51(\mathrm{~s}, 3 \mathrm{H}), 2.55-2.56(\mathrm{~m}, 1 \mathrm{H}), 2.73-2.81(\mathrm{~m}, 1 \mathrm{H}), 3.77-3.83(\mathrm{~m}, 1 \mathrm{H}), 4.09-4.18(\mathrm{~m}, 1 \mathrm{H})$ 7.29-7.35 (m, 2H), $7.49(\mathrm{~d}, J=7.7 \mathrm{~Hz}, 2 \mathrm{H}), 7.56-7.62(\mathrm{~m}, 2 \mathrm{H}), 7.93(\mathrm{~d}, J=8.3 \mathrm{~Hz}, 2 \mathrm{H})$. ${ }^{13} \mathrm{C}$ NMR (101 MHz, MeOD): $\delta=21.5\left(\mathrm{CH}_{3}\right), 22.5\left(\mathrm{CH}_{3}\right), 33.8\left(\mathrm{CH}_{2}\right), 37.5\left(\mathrm{CH}_{2}\right), 81.0$, $83.5,87.4,113.4,125.0,125.4,127.8,130.6,130.6,131.7,140.0,142.6,143.4,145.2$, $170.1(\mathrm{C}=\mathrm{O}) .{ }^{19} \mathrm{~F}$ NMR $(376 \mathrm{MHz}, \mathrm{MeOD}): \delta=-77.5 . \mathrm{IR} v\left(\mathrm{~cm}^{-1}\right): 3306(\mathrm{~N}-\mathrm{H}), 2921(\mathrm{Ar}-$ 
H), 2851 (Ar-H), 1599 (C=O), 1469, 1279, 1138, 1082, 753, 666, 547. HRMS (ESI-TOF): calcd. for $\mathrm{C}_{19} \mathrm{H}_{22} \mathrm{~N}_{3} \mathrm{O}_{4} \mathrm{~S}: 388.1326$; found: 388.1311 .

\section{2-(3-(Benzoyloxy)-2-(tosylimino)indolin-3-yl)ethanaminium 2,2,2-trifluoroacetate 17b.}

$67 \mathrm{mg}, 99 \%$ yield, yellow solid. m.p. $=131-135{ }^{\circ} \mathrm{C} .{ }^{1} \mathrm{H}$ NMR $\left(400 \mathrm{MHz}, \mathrm{CDCl}_{3}\right): \delta=2.19-$ $2.23(\mathrm{~m}, 1 \mathrm{H}), 2.43(\mathrm{~s}, 3 \mathrm{H}), 3.61-3.64(\mathrm{~m}, 1 \mathrm{H}), 3.74(\mathrm{td}, J=7.1,6.9,2 \mathrm{H}), 5.38(\mathrm{~s}, 1 \mathrm{H}), 7.07$ (d, $J=8.1 \mathrm{~Hz}, 1 \mathrm{H}), 7.18-7.54(\mathrm{~m}, 8 \mathrm{H}), 7.74(\mathrm{~d}, J=7.6 \mathrm{~Hz}, 2 \mathrm{H}), 7.88(\mathrm{~d}, J=8.0 \mathrm{~Hz}, 2 \mathrm{H})$. ${ }^{13} \mathrm{C}$ NMR $\left(101 \mathrm{MHz}, \mathrm{CDCl}_{3}\right): \delta=21.6\left(\mathrm{CH}_{3}\right), 34.8\left(\mathrm{CH}_{2}\right), 37.4\left(\mathrm{CH}_{2}\right), 86.6,111.7,124.6$, $126.5,126.6,127.0,128.5,128.6,129.3,129.7,130.8,131.8,138.0,144.0,168.2(\mathrm{C}=\mathrm{O})$.

${ }^{19}$ F NMR (376 MHz, $\left.\mathrm{CDCl}_{3}\right): \delta=-76.0 . \mathrm{IR} v\left(\mathrm{~cm}^{-1}\right): 3253(\mathrm{~N}-\mathrm{H}), 3068(\mathrm{Ar}-\mathrm{H}), 2920(\mathrm{Ar}-$ H), $1627(\mathrm{C}=\mathrm{O})$, 1550, 1381, 1167, 876, 657, 540. HRMS (ESI-TOF): calcd. for $\mathrm{C}_{24} \mathrm{H}_{24} \mathrm{~N}_{3} \mathrm{O}_{4} \mathrm{~S}: 450.1482$; found: 450.1476 .

\section{2-(3-Acetoxy-1-methyl-2-(tosylimino)indolin-3-yl)ethanaminium 2,2,2-trifluoroacetate}

18a. $61 \mathrm{mg}, 99 \%$ yield, yellow solid. m.p. $=129-132{ }^{\circ} \mathrm{C} .{ }^{1} \mathrm{H} \mathrm{NMR}\left(400 \mathrm{MHz}, \mathrm{CDCl}_{3}\right): \delta=$ 2.19-2.23 (m, 1H), $2.46(\mathrm{~s}, 3 \mathrm{H}), 2.55(\mathrm{~s}, 3 \mathrm{H}), 3.20-3.28(\mathrm{~m}, 1 \mathrm{H}), 3.48(\mathrm{~s}, 3 \mathrm{H}), 3.84-3.95(\mathrm{~m}$, $1 \mathrm{H}), 4.04-4.11(\mathrm{~m}, 1 \mathrm{H}), 7.05(\mathrm{~d}, J=7.9 \mathrm{~Hz}, 1 \mathrm{H}), 7.26-7.30(\mathrm{~m}, 1 \mathrm{H}), 7.36(\mathrm{~d}, J=8.0 \mathrm{~Hz}$, 2H), $7.44(\mathrm{dd}, J=7.6,1.1 \mathrm{~Hz}, 1 \mathrm{H}), 7.55(\mathrm{td}, J=7.9,1.1 \mathrm{~Hz}, 1 \mathrm{H}), 7.83-7.96(\mathrm{~m}, 2 \mathrm{H}) .{ }^{13} \mathrm{C}$ $\operatorname{NMR}\left(101 \mathrm{MHz}, \mathrm{CDCl}_{3}\right): \delta=20.0\left(\mathrm{CH}_{3}\right), 21.5\left(\mathrm{CH}_{3}\right), 25.1\left(\mathrm{CH}_{3}\right), 30.4\left(\mathrm{CH}_{2}\right), 34.5\left(\mathrm{CH}_{2}\right)$, $84.3,110.5,123.7,125.3,126.3,127.1,129.6,132.9,138.9,142.5,143.8,162.5,172.8$ $(\mathrm{C}=\mathrm{O}) .{ }^{19} \mathrm{~F}$ NMR $\left(376 \mathrm{MHz}, \mathrm{CDCl}_{3}\right): \delta=-75.9 . \mathrm{IR} v\left(\mathrm{~cm}^{-1}\right): 3383(\mathrm{~N}-\mathrm{H}), 2919(\mathrm{Ar}-\mathrm{H})$, $1678(\mathrm{C}=\mathrm{O}), 1572,1190,1148,1132,1083,948,777,760,680$. HRMS (ESI-TOF): calcd. for $\mathrm{C}_{20} \mathrm{H}_{24} \mathrm{~N}_{3} \mathrm{O}_{4} \mathrm{~S}$ : 402.1488; found: 402.1485 . 
trifluoroacetate 18b. $89 \mathrm{mg}, 99 \%$ yield, yellow solid. m.p. $=56-58{ }^{\circ} \mathrm{C} .{ }^{1} \mathrm{H}$ NMR $(400$ $\left.\mathrm{MHz}, \mathrm{CDCl}_{3}\right): \delta=2.31-2.34(\mathrm{~m}, 1 \mathrm{H}), 2.39(\mathrm{~s}, 3 \mathrm{H}), 3.27-3.34(\mathrm{~m}, 1 \mathrm{H}), 3.53(\mathrm{~s}, 3 \mathrm{H}), 4.09-$ $4.14(\mathrm{~m}, 1 \mathrm{H}), 4.21-4.28(\mathrm{~m}, 1 \mathrm{H}), 7.06(\mathrm{~d}, J=8.0 \mathrm{~Hz}, 1 \mathrm{H}), 7.21-7.28(\mathrm{~m}, 3 \mathrm{H}), 7.50-7.55(\mathrm{~m}$

4H), 7.70-7.71 (m, 3H), 8.00-8.08 (m, 2H). $\left.{ }^{13} \mathrm{C} \mathrm{NMR} \mathrm{(101} \mathrm{MHz,} \mathrm{CDCl}_{3}\right): \delta=21.5\left(\mathrm{CH}_{3}\right)$, $25.4\left(\mathrm{CH}_{3}\right), 30.7\left(\mathrm{CH}_{2}\right), 35.1\left(\mathrm{CH}_{2}\right), 84.7,110.6,124.0,124.9,125.4,126.3,127.0,128.9$, 129.4, 129.5, 132.5, 135.9, 138.8, 142.6, 143.7, 162.2, $167.6(\mathrm{C}=\mathrm{O}) .{ }^{19} \mathrm{~F}$ NMR $(376 \mathrm{MHz}$ $\left.\mathrm{CDCl}_{3}\right): \delta=-75.9 . \mathrm{IR} v\left(\mathrm{~cm}^{-1}\right): 3067(\mathrm{~N}-\mathrm{H}), 2929(\mathrm{Ar}-\mathrm{H}), 1783(\mathrm{C}=\mathrm{O}), 1588,1138,1084$ HRMS (ESI-TOF): calcd. for $\mathrm{C}_{25} \mathrm{H}_{25} \mathrm{~N}_{3} \mathrm{NaO}_{4} \mathrm{~S}$ : 486.1463; found: 486.1458 .

\section{N-(3-(2-Acetamidoethyl)-3-hydroxy-1-methylindolin-2-ylidene)-4- methylbenzenesulfonamide 19}

Compound 18a (46 mg, $0.1 \mathrm{mmol})$ was dissolved in $\mathrm{CH}_{2} \mathrm{Cl}_{2}(0.25 \mathrm{~mL})$ and $\mathrm{Et}_{3} \mathrm{~N}(0.5 \mathrm{~mL})$ was added dropwise. After 16 hours, additional $\mathrm{CH}_{2} \mathrm{Cl}_{2}(5 \mathrm{~mL})$ and water were added, and the resulting mixture was extracted with diluted $\mathrm{HCl}(1 \mathrm{M}$ in water). The organic phase was separated and the solvent was removed under reduced pressure. The title compound was isolated as a yellowish solid $(40 \mathrm{mg}, 0.1 \mathrm{mmol}, 99 \%$ yield $) . \mathrm{m} . \mathrm{p} .=135-138{ }^{\circ} \mathrm{C} .{ }^{1} \mathrm{H} \mathrm{NMR}$ $\left(400 \mathrm{MHz}, \mathrm{CDCl}_{3}\right): \delta=1.90(\mathrm{~s}, 3 \mathrm{H}), 2.45(\mathrm{~s}, 3 \mathrm{H}), 3.19(\mathrm{~s}, 3 \mathrm{H}), 2.45-2.55(\mathrm{~m}, 1 \mathrm{H}), 2.94-$ $3.17(\mathrm{~m}, 3 \mathrm{H}), 3.19(\mathrm{~s}, 3 \mathrm{H}), 6.31$ (brs, $1 \mathrm{H}), 6.90(\mathrm{~d}, J=8.0 \mathrm{~Hz}, 1 \mathrm{H}), 7.19-7.23(\mathrm{~m}, 1 \mathrm{H})$, 7.32-7.34 (m, 2H), $7.35-7.41(\mathrm{~m}, 2 \mathrm{H}), 7.88-7.90(\mathrm{~m}, 2 \mathrm{H}), 8.08-8.10(\mathrm{~m}, 1 \mathrm{H}) .{ }^{13} \mathrm{C} \mathrm{NMR}$ $\left(101 \mathrm{MHz}, \mathrm{CDCl}_{3}\right): \delta=21.6\left(\mathrm{CH}_{3}\right), 23.1\left(\mathrm{CH}_{3}\right), 28.4\left(\mathrm{CH}_{3}\right), 34.5\left(\mathrm{CH}_{2}\right), 38.8\left(\mathrm{CH}_{2}\right), 80.2$ (C-O), 109.8, 123.4, 125.0, 126.4, 129.4, 129.7, 130.4, 130.5, 139.1, 142.7, 143.3, 169.9, $171.2(\mathrm{C}=\mathrm{O})$. IR $v\left(\mathrm{~cm}^{-1}\right): 3411(\mathrm{O}-\mathrm{H}), 3376(\mathrm{~N}-\mathrm{H}), 2921(\mathrm{Ar}-\mathrm{H}), 2852(\mathrm{Ar}-\mathrm{H}), 1665$ 
$(\mathrm{C}=\mathrm{O}), \quad 1548, \quad 1073, \quad 961, \quad 778, \quad 752, \quad 689$, 553. HRMS (ESI-TOF): calcd. for $\mathrm{C}_{20} \mathrm{H}_{23} \mathrm{~N}_{3} \mathrm{NaO}_{4} \mathrm{~S}: 424.1301$; found: 424.1307.

8-Methyl-2,3,3a,8-tetrahydropyrrolo[2,3-b]indol-3a-yl benzoate 20. Compound $18 b$ (0.372 g, 0.64 mmol, 1 equiv) was dissolved in $\mathrm{MeOH}(10 \mathrm{~mL})$ and $\mathrm{LiOH}(0.154 \mathrm{~g}, 6.4$ mmol, 10 equiv) was added. The reaction mixture was allowed to stir at $25^{\circ} \mathrm{C}$ for $16 \mathrm{~h}$. The solvent was removed under reduced pressure. The residue was quenched with water and extracted with $\mathrm{CH}_{2} \mathrm{Cl}_{2}$. The organic phase was dried over $\mathrm{Na}_{2} \mathrm{SO}_{4}$, filtered and the solvent was removed under reduced pressure affording compound $\mathbf{2 0}$ in quantitative yield (185 $\mathrm{mg}$, 99\%) as a brownish solid. m.p. $=45-46{ }^{\circ} \mathrm{C} .{ }^{1} \mathrm{H}$ NMR (500 MHz, $\left.\mathrm{CDCl}_{3}\right): \delta=2.07$ (ddd, $J=$ 14.0, 7.1, $5.3 \mathrm{~Hz}, 1 \mathrm{H}), 2.23$ (ddd, $J=14.0,6.5,5.3 \mathrm{~Hz}, 1 \mathrm{H}), 3.27$ (s, 3H), 3.90 (ddd, $J=$ 17.2, 6.5, 5.3 Hz, 1H), 4.17 (ddd, $J=17.2,7.1,5.3 \mathrm{~Hz}, 1 \mathrm{H}), 6.92$ (d, $J=7.9 \mathrm{~Hz}, 1 \mathrm{H}), 7.13$ $(\mathrm{t}, J=7.6 \mathrm{~Hz}, 1 \mathrm{H}), 7.34-7.44(\mathrm{~m}, 5 \mathrm{H}), 7.91-7.94(\mathrm{~m}, 2 \mathrm{H}) .{ }^{13} \mathrm{C} \mathrm{NMR}\left(125 \mathrm{MHz}, \mathrm{CDCl}_{3}\right): \delta$ $=26.4\left(\mathrm{CH}_{3}\right), 27.5\left(\mathrm{CH}_{2}\right), 38.7\left(\mathrm{CH}_{2}\right), 76.7,108.7,123.4,124.1,127.2,128.0,129.1,130.4$, 130.5, 133.5, 143.2, 154.3, $174.2(\mathrm{C}=\mathrm{O})$. IR $v\left(\mathrm{~cm}^{-1}\right): 3060(\mathrm{Ar}-\mathrm{H}), 2924(\mathrm{Ar}-\mathrm{H}), 1715$ $(\mathrm{C}=\mathrm{O}), 1653,1613,1156,1120,1091$. HRMS (ESI-TOF): calcd. for $\mathrm{C}_{18} \mathrm{H}_{17} \mathrm{~N}_{2} \mathrm{O}_{2}$ : 293.1285; found: 293.1284 .

\section{Trifluoromethyl 3-(2-benzamidoethyl)-1-methyl-2-oxoindoline-3-carboxylate 21.}

Compound 20 (58 mg, 0.20 mmol, 1 equiv) was dissolved in $\mathrm{CH}_{2} \mathrm{Cl}_{2}(2 \mathrm{~mL})$ and TFA (0.05 $\mathrm{mL}, 0.60 \mathrm{mmol}, 3$ equiv) was added. The reaction mixture was stirred for $16 \mathrm{~h}$ at $25{ }^{\circ} \mathrm{C}$. After 16 hours, the solvent was removed under reduced pressure to afford the pure product as a yellow oil in $80 \mathrm{mg}\left(99 \%\right.$ yield). ${ }^{1} \mathrm{H}$ NMR $\left(400 \mathrm{MHz}, \mathrm{CDCl}_{3}\right): \delta=2.45-2.50(\mathrm{~m}, 1 \mathrm{H})$, 2.68-2.74 (m, 1H), $3.29(\mathrm{~s}, 3 \mathrm{H}), 4.09-4.14(\mathrm{~m}, 1 \mathrm{H}), 4.40-4.47(\mathrm{~m}, 1 \mathrm{H}), 7.04(\mathrm{~d}, J=7.9$, 
1H), 7.26 (t, $J=8.0 \mathrm{~Hz}, 1 \mathrm{H}), 7.36-7.59(\mathrm{~m}, 3 \mathrm{H}), 7.74(\mathrm{t}, J=7.4 \mathrm{~Hz}, 1 \mathrm{H}), 7.67-7.77(\mathrm{~m}$,

1H), $7.98(\mathrm{~d}, J=7.3 \mathrm{~Hz}, 2 \mathrm{H}) .{ }^{13} \mathrm{C}$ NMR $\left(101 \mathrm{MHz}, \mathrm{CDCl}_{3}\right): \delta=25.0\left(\mathrm{CH}_{3}\right), 26.7\left(\mathrm{CH}_{2}\right)$, $35.8\left(\mathrm{CH}_{2}\right), 81.4,109.9,112.0,114.3,116.5,118.8,123.7,124.0,125.0,128.4,129.5$, 132.8, 136.2, 143.5, $161.3\left(\mathrm{q}, \mathrm{CF}_{3} \mathrm{C}=\mathrm{O}\right), 168.4(\mathrm{C}=\mathrm{O}), 170.8(\mathrm{C}=\mathrm{O}) .{ }^{19} \mathrm{~F}$ NMR $(101 \mathrm{MHz}$, $\left.\mathrm{CDCl}_{3}\right): \delta=-76.0 . \mathrm{IR} v\left(\mathrm{~cm}^{-1}\right): 3325(\mathrm{~N}-\mathrm{H}), 2926(\mathrm{Ar}-\mathrm{H}), 1697(\mathrm{C}=\mathrm{O}), 1638(\mathrm{C}=\mathrm{O}), 1611$ $(\mathrm{C}=\mathrm{O}), 1537,1490,1347,1301,1128,1091,749,695$. HRMS (ESI-TOF): calcd. for $\mathrm{C}_{20} \mathrm{H}_{17} \mathrm{~F}_{3} \mathrm{~N}_{2} \mathrm{NaO}_{2}$ : 429.1038, found: 429.1026 .

\section{Supporting Information}

Reproduction of NMR spectra for new compounds and ORTEP plots from X-ray analyses (pdf format), and individual $X$-ray crystallographic data for compounds $9, \mathbf{1 1} / \mathbf{1 2}, \mathbf{1 4 a}, \mathbf{1 8 a}$, 19, and 20, respectively (cif). This material is available free of charge via the Internet at http://pubs.acs.org.

\section{Acknowledgements}

Financial support for this project was provided from the Spanish Ministry for Economy and Competitiveness and FEDER (CTQ2014-56474R grant to K. M., and Severo Ochoa Excellence Accreditation 2014-2018 to ICIQ, SEV-2013-0319). A.E.B. acknowledges a FPI fellowship and C. M. support from Cellex Foundation. The authors thank E. EscuderoAdán for the X-ray structural analyses.

\section{References}

(1) Zhdankin, V. V. Hypervalent Iodine Chemistry Preparation, Structure and Synthetic Applications of Polyvalent Iodine Compounds, Wiley, Chichester 2013. 
(2) (a) Hypervalent Iodine Chemistry. Modern Developments in Organic Synthesis, Wirth, T. (Ed.), Top. Curr. Chem. 224, Springer, Berlin, 2003. (b) Yoshimura, A.; Zhdankin, V. V. Chem. Rev. 2016, 116, 3328. (c) Richardson, R. D.; Wirth, T. Angew. Chem. Int. Ed. 2006, 45, 4402. (d) Ochiai, M. Chem. Rec. 2007, 7, 12. (e) Dohi, T.; Kita, Y. Chem. Commun. 2009, 2073. (f) Uyanik, M.; Ishihara, K. Chem. Commun. 2009, 2086.

(3) (a) Fra, L.; Millán, A.; Souto, J. A.; K. Muñiz, K. Angew. Chem. Int. Ed. 2014, 53, 7349. (b) Fra, L.; Muñiz, K. Chem. Eur. J. 2016, 22, 4351.

(4) (a) Liu, K.; Wen, P.; Liu, J.; Huang, G. Synthesis 2010, 3623. (b) Liu, Q.; Li, G.; Yi, H.; Wu, P.; Liu, J.; Lei, A. Chem. Eur. J. 2011, 17, 2353. (c) Soni, V.; Patel, U. N.; Punji, B. RSC Adv. 2015, 5, 57472.

(5) Liu, Q.; Zhao, Q. Y.; Liu, J.; Wu, P.; Yi, H.; Lei, A. Chem. Commun. 2012, 48, 3239.

(6) Ackermann, L.; Dell'Acqua, M.; Fenner, S.; Vicente, R.; Sandmann, R. Org. Lett. 2011, $13,2358$.

(7) Souto, J. A.; Martínez, C.; Velilla, I.; Muñiz, K. Angew. Chem. Int. Ed. 2013, 52, 1324.

(8) For related bromine(III) reagents incorporating transferable nitrogen groups: (a) Ochiai, M.; Kaneaki, T.; Tada, N.; Miyamoto, K.; Chuman, H.; Shiro, M.; Hayashi, S.; Nakanishi, W. J. Am. Chem. Soc. 2007, 129, 12938. (b) Ochiai, M.; Miyamoto, K.; Kaneaki, T.; Hayashi, S.; Nakanishi, W. Science 2011, 332, 448.

(9) For the alternative concept of electrophilic amination with hypervalent iodine reagents:

(a) Tellitu, I.; Domínguez, E. Trends Heterocycl. Chem. 2011, 15, 23. (b) Narayan, R.; Manna, S.; Antonchick, A. P. Synlett 2015, 26, 1785. (c) Samanta, R.; Matcha, K.; Antonchick, A. P. Eur. J. Org. Chem. 2013, 5769.

(10) (a) Yoshimura, A.; Koski, S. R.; Fuchs, J. M.; Saito, A.; Nemykin, V. N.; Zhdankin, V. V. Chem. Eur. J. 2015, 21, 5328. (b) Kiyokawa, K.; Kosaka, T.; Kojima, T.; Minakata, 
S. Angew. Chem. Int. Ed. 2015, 54, 13719. (c) Hadjiarapoglou, L.; Spyroudis, S.; Varvoglis, A. Synthesis 1983, 207. (d) Papadopoulou, M.; Varvoglis, A. Chem. Res. Synop. 1983, 66. (e) Papadopoulou, M.; Varvoglis, A. Chem. Res. Synop. 1984, 166.

(11) Souto, J. A.; Zian, D.; Muñiz, K. J. Am. Chem. Soc. 2012, 134, 7242.

(12) Kiyokawa, K.; Yahata, S.; Kojima, T.; Minakata, S. Org. Lett. 2014, 16, 4646.

(13) Souto, J. A.; Becker, P.; Iglesias, A.; Muñiz, K. J. Am. Chem. Soc. 2012, 134, 15505.

(14) Purkait, N.; Okamura, S.; Souto, J. A.; Muñiz, K. Org. Lett. 2014, 16, 4750.

(15) Moriyama, K.; Ishid, K.; Togo, H. Chem. Commun. 2015, 51, 2273.

(16) (a) Souto, J. A.; González, Y.; Iglesias, Á.; Zian, D.; Lishchynskyi, A.; Muñiz, K. Chem. Asian J. 2012, 7, 1103. (b) Röben, C.; Souto, J. A.; González, Y.; Lishchynskyi, A.; Muñiz, K. Angew. Chem. Int. Ed. 2011, 50, 9478. (c) Röben, C.; Souto, J. A.; EscuderoAdán, E. C. Muñiz, K. Org. Lett. 2013, 15, 1008.

(17) Lishchynskyi, A.; Muñiz, K. Chem. Eur. J. 2012, 18, 2213.

(18) For an overview on alkene difunctionalization with hypervalent iodine(III) reagents: Romero, R. M.; Wöste, T. H.; Muñiz, K. Chem. Asian J. 2014, 9, 972.

(19) Liu, H.-H.; Wang, Y.; Deng, G.; Yang, L. Adv. Synth. Catal. 2013, 355, 3369.

(20) (a) Li, Y.-X.; Ji, K.-G.; Wang, H.-X.; Ali, S.; Liang, Y.-M. J. Org. Chem. 2011, 76, 744. (b) Li, Y.-X.; Wang, H.-X.; Ali, S.; Xia, X.-F.; Liang, Y.-M. Chem. Commun. 2012, 48, 2343. (c) Wu, W.-B.; Huang, J.-M. Org. Lett. 2012, 14, 5832. (d) Ali, A.; Li, Y.-X.; Anwar, S.; Yang, F.; Chen, Z.-S.; Liang, Y.-M. J. Org. Chem. 2012, 77, 424. (e) Prasad, B.; Sreenivas, B. Y.; Rambabu, D.; Krishna, G. R.; Reddy, C. M.; Kumar, K. L.; Pal, M. Chem. Commun. 2013, 49, 3970.

(21) Moriyama, K.; Ishid, K.; Togo, H. Chem. Commun. 2015, 51, 2273. 
(22) For the same concept using subsequent copper catalysis for 3-amination of indoles: (a) Sokolovs, I.; Lubriks, D.; Suna, E. J. Am. Chem. Soc. 2012, 134, 15436. (b) Lubriks, D.; Sokolovs, I.; Suna, E. J. Am. Chem. Soc. 2014, 136, 6920.

(23) For recent other, mechanistically non-related 2-amination reactions, see: (a) Shi, J.; Zhou, B.; Yang, Y.; Li, Y. Org. Biomol. Chem. 2012, 10, 8953. (b) Qin, Q.; Yu, S. Org. Lett. 2014, 16, 3504. (c) Greulich, T. W.; Daniliuc, C. G.; Studer, A. Org. Lett. 2015, 17, 254. (d) Brachet, E.; Ghosh, T.; Ghosh, I.; König, B. Chem. Sci. 2015, 6, 987.

(24) (a) Steven, A.; Overman, L. E. Angew. Chem. Int. Ed. 2007, 46, 5488. (b) Crich, D.; Banerjee, A. Acc. Chem. Res. 2007, 40, 151. (c) Ruiz-Sanchis, P.; Savina, S. A.; Albericio, F.; Álvarez, M. Chem. Eur. J. 2011, 17, 1388.

(25) (a) Kajiyama, D.; Saitoh, T.; Yamaguchi, S.; Nishiyama, S. Synthesis 2012, 44, 1667. (b) Yang, Y.; Jiang, X.; Qing, F.-L. J. Org. Chem. 2012, 77, 7538.

(26) (a) Barluenga, J. Pure Appl. Chem. 1999, 71, 431. (b) Barluenga, J.; González, J. M.; Campos, P. J.; Asensio, G. Angew. Chem. Int. Ed. 1985, 24, 319. (c) Barluenga, J.; González-Bobes, F.; Murguía, M. C.; Ananthoju, S. R.; González, J. M. Chem. Eur. J. 2004, 10, 4206. (d) Espuña, G.; Ananthoju, S. R.; Andreu, D.; Barluenga, J.; Pérez, X.; Planas, A.; Arsequell, G.; Valencia, G. Biochem. 2006, 45, 5957. (e) Barluenga, J.; González, J. M.; García-Martín, M. A.; Campos, P. J.; Asensio, G. J. Org. Chem. 1993, 58, 2058.

(27) See Supporting Information for details and X-ray analyses.

(28) (a) Loach, R. P.; Fenton, O. S.; Amaike, K.; Siegel, D. S.; Movassaghi, M. J. Org. Chem. 2014, 79, 11254 and cited literature. For enzymatic approaches: (b) Wiesner, W.; Vanpee, K. H.; Lingens, F. FEBS Lett. 1986, 209, 321. (c) Dong, C. J.; Flecks, S.; Unversucht, S.; Haupt, C.; van Pee, K. H.; Naismith, J. H. Science 2005, 309, 2216. 
(29) (a) Lozano, O.; Blessley, G.; Martínez del Campo, T.; Thompson, A. L.; Giuffredi, G. T.; Bettati, M.; Walker, M.; Borman, R.; Gouverneur, V. Angew. Chem. Int. Ed. 2011, 50, 8105. (b) Tréguier, B.; Roche, S. P. Org. Lett. 2014, 16, 278.

(30) (a) Peddibhotla, S. Curr. Bioact. Comp. 2009, 5, 20. (b) Yan, T.; Wang, X.; Sun, H.; Liu, J.; Xie, Y. Molecules 2013, 18, 14505.

(31) Müller, K.; Faeh, C.; Diederich, F. Science 2007, 317, 1881.

(32) Anumadla, D.; Jeffrey, C. S. J. Am. Chem. Soc. 2015, 137, 14858.

(33) Song, H.; Yang, J.; Chen, W.; Gin, Y. Org. Lett. 2006, 8, 6011.

(34) Desaty, D.; Keglevic, D. Croatica Chem. Acta 1965, 37, 25.

(35) Nicolaou, K. C.; Krasovskiy, A.; Majumder, Trepanier, V. E.; Chen, D. Y.-K. J. Am. Chem. Soc. 2009, 131, 3690. 